\title{
SUBMISSION TO GÉOTECHNIQUE
}

\section{DATE:}

$28 / 03 / 2018$

\section{TITLE:}

The parkable piezoprobe for determining $\mathrm{c}_{\mathrm{v}}$ and strength - modelling and interpretation methods

\section{AUTHOR:}

Schneider, M.A. ${ }^{1}$, Stanier, S.A. ${ }^{2}$, Chatterjee, S. ${ }^{3}$, White, D.J. ${ }^{4}$ and Randolph, M.F. ${ }^{5}$

\section{POSITION AND AFFILIATION:}

${ }^{1}$ Research Student at the Centre for Offshore Foundation Systems, University of Western Australia

${ }^{2}$ ARC DECRA Fellow at the Centre for Offshore Foundation Systems, University of Western Australia

${ }^{3}$ Assistant Professor, Department of Civil Engineering, Indian Institute of Technology Bombay, India

${ }^{4}$ Professor, University of Southampton (formerly University of Western Australia)

${ }^{5}$ Professor at the Centre for Offshore Foundation Systems, University of Western Australia

\section{CONTACT ADDRESS:}

Dr Sam Stanier

Centre for Offshore Foundation Systems

The University of Western Australia (M053)

35 Stirling Highway

Crawley, Perth

WA 6009, Australia

\section{NUMBER OF WORDS, FIGURES AND TABLES:}

Words: 5612

Figures: 14

Tables: 6

\section{KEYWORDS:}

Dissipation; consolidation; offshore engineering; in situ testing; centrifuge modelling; finite element analysis 
Schneider, M. A., Stanier, S. A., Chatterjee, S., White, D. J. and Randolph, M. F.

The parkable piezoprobe for determining $c_{v}$ and strength - modelling and interpretation methods

\title{
THE PARKABLE PIEZOPROBE FOR DETERMINING Cv AND STRENGTH - MODELLING AND INTERPRETATION METHODS
}

\author{
Schneider, M.A., Stanier, S.A., Chatterjee, S., White, D.J. and Randolph, M.F.
}

\section{ABSTRACT}

The parkable piezoprobe (PPP) is a site investigation tool for measuring the coefficient of consolidation, in-situ offshore, at shallow embedment depths. The device applies a similar bearing pressure to subsea infrastructure so reaches a comparable self-weight penetration when 'parked' at the seabed, representative of an unburied pipeline. Instrumentation on the device allows the dissipation of penetration-induced excess pore pressure to be recorded at various locations on the surface. From these dissipation responses the coefficient of consolidation can be inferred, which is a key parameter in the design of many offshore structures founded in surficial soil, such as pipelines or shallow foundations. The intent is that this device is deployed from a seabed frame while other activities such as penetrometer testing or sampling take place in parallel.

This paper presents robust interpretation methods for the parkable piezoprobe by using a combination of centrifuge experiments and large deformation finite element analyses. The centrifuge tests demonstrate that the penetration response of the parkable piezoprobe is adequately captured by existing bearing capacity models, allowing the optimum device weight to be identified. A comprehensive interpretation method is then developed for the dissipation stage. This yields accurate estimates of the coefficient of consolidation, even for cases where there is no prior knowledge of the soil parameters or the depth to which the device embeds under its own self-weight. 
Schneider, M. A., Stanier, S. A., Chatterjee, S., White, D. J. and Randolph, M. F.

The parkable piezoprobe for determining $c_{v}$ and strength - modelling and interpretation methods

\section{INTRODUCTION}

Offshore infrastructure to extract hydrocarbons or develop renewable energy (e.g. pipelines, cables or shallow foundations) is increasingly founded in regions with very soft surficial soils. Robust design of these structures requires reliable measurements of the soil properties, such as undrained strength, $s_{u}$, and coefficient of consolidation, $c_{v}$. Conventional tools such as the cone penetrometer (Teh \& Houlsby, 1991), piezoprobe (Whittle et al. 2001) and Piezoball (Colreavy et al. 2015) are not suited to the very shallow embedment depths relevant to pipeline design. Specialist tools such as actuator-controlled instrumented model pipes (Hill \& Jacob 2008; White et al. 2011) are able to measure $c_{v}$ at the shallow depths required but are prohibitively expensive to routinely deploy.

The parkable piezoprobe (PPP) - as described by Chatterjee et al. (2014) and illustrated in Figure 1 - is a device intended primarily to measure the coefficient of consolidation, $c_{v}$. The main benefit of the device is that it does not have to be attached to a drill string or a set of push rods during operation (when 'parked'), making deployment simple and allowing other activities to be performed in parallel, before retrieving the probe. This is a valuable feature since in-situ dissipation tests are costly to perform offshore, due to the time occupied waiting. The ability to run PPP tests unmonitored allows the device to be of similar scale to the infrastructure intended to be installed at the location (e.g. similar to the diameter of a seabed pipeline).

Chatterjee et al. (2014) first described the PPP and reported non-dimensional dissipation solutions for a limited range of soil conditions derived from large deformation finite element analyses. This paper first presents experimental modelling of the PPP concept via small-scale centrifuge model tests. The centrifuge experiments are used to validate a large deformation finite element model, which is then harnessed in an extensive parametric study exploring the sensitivity of the PPP response to the range of soil conditions likely to be found offshore in regions with soft fine-grained surficial soil. Finally, a simple interpretation method is 
proposed that could be used to interpret measurements derived from field deployment of a PPP, which results in accurate estimates of the coefficient of consolidation.

\section{PARKABLE PIEZOPROBE}

The parkable piezoprobe (PPP) is an elongated spheroid with pore pressure transducers located at the invert and midface positions (see Figure 1). The cross-section of the shape (in elevation) and its diameter (at prototype scale) are comparable to a small seabed pipeline. The probe is envisaged to be lowered on a cable winch from a vessel or from a seabed frame. Alternatively, it could also be deployed from a remotely-operated vehicle (ROV).

After the initial undrained penetration stage, which generates excess pore pressures in the soil, the dissipation response is recorded by an on-board logging system. With suitable interpretation methods this data can be used to estimate the coefficient of consolidation. More accurate estimates can be gained if the settlement is also measured during penetration, as is demonstrated later in the paper. The embedment depth could be estimated via additional pore pressure transducers along the surface of the $\mathrm{PPP}^{1}$, so that soil contact would be indicated by an initial spike in the corresponding pore water pressure reading. Alternatively, contact image sensors (CIS) could be installed on the surface of the device and used to estimate the soil contact (An et al. 2016).

A probe diameter of $250 \mathrm{~mm}$ is suggested for field application of the device. The following discussion uses the initial dissipation solutions of Chatterjee et al. (2014) to illustrate the practical timescale of a PPP test for measuring dissipation characteristics, i.e. the coefficient of consolidation (referred to as $c_{v}$ for a conventional oedometer or Rowe cell test and $c_{h}$ for a cone penetrometer or pipe section test) relative to other available devices.

\footnotetext{
${ }^{1}$ This was not possible on the centrifuge scale model developed for this study but would be possible on a larger field scale device.
} 
Schneider, M. A., Stanier, S. A., Chatterjee, S., White, D. J. and Randolph, M. F.

The parkable piezoprobe for determining $c_{v}$ and strength - modelling and interpretation methods

The time required for $50 \%$ of the excess pore pressure at the invert of a $250 \mathrm{~mm}$ PPP to dissipate, $t_{50}$, is between 2.9 and 4.4 times that of a standard cone penetrometer $(D=35.7 \mathrm{~mm})$ for an embedment of $w / D=0.5$ and $w / D=1$, respectively. Although this testing time exceeds the CPT $t_{50}$, the PPP is uniquely able to measure $c_{v}$ at very shallow depths where the conventional CPT dissipation interpretation is not reliable. The only other device suited to shallow dissipation characteristics determination is the SMARTPIPE (Hill \& Jacob 2008; White et al. 2011). This pipe-like site investigation tool has dissipation times between 1.5 and 2.3 times longer than the PPP, even though the device diameters are similar (PPP: $250 \mathrm{~mm}$; SMARTPIPE: $225 \mathrm{~mm}$ ). This is due to the planar drainage around the SMARTPIPE. The radial drainage paths around the PPP $(D=250 \mathrm{~mm})$ result in shorter test durations despite its slightly larger diameter. Figure 2 shows the time required for $50 \%$ of the excess pore pressure to dissipate for different values of the coefficient of consolidation, for the PPP, cone penetrometer and SMARTPIPE. The comparison is based on the following dimensionless dissipation time $T_{50}=c_{\nu} t_{50} / D^{2}$ : for the PPP, $T_{50}=0.036$ for $w / D=0.5$ and $T_{50}=0.055$ for $w / D=1$ (Chatterjee et al., 2014); for the cone penetrometer $T_{50}=0.613$ for $I_{R}=100$ (Teh \& Houlsby, 1991); and for the SMARTPIPE $T_{50}=0.10$ (Gourvenec \& White, 2010).

\section{CENTRIFUGE MODELLING}

A total of four centrifuge tests of the PPP were carried out in the geotechnical beam centrifuge at UWA. In each test an initial undrained penetration to a target depth of either $0.5 D$ or $1 D$ (achieved using the actuator in displacement controlled (DC) mode) was followed by a pore pressure dissipation stage (achieved using the actuator in load controlled (LC) mode using feedback from the load cell). During the consolidation phase either the vertical load (LC: load controlled mode) or the displacement (DC: displacement controlled mode) was held constant. The LC mode represents the PPP resting in equilibrium with the self-weight 
Schneider, M. A., Stanier, S. A., Chatterjee, S., White, D. J. and Randolph, M. F.

The parkable piezoprobe for determining $c_{v}$ and strength - modelling and interpretation methods

balanced by the seabed bearing capacity, although in practice the device was fixed to an actuator under feedback control. All model tests are summarized in Table 1.

Table 1: Summary of experimental tests. LC: Load control; DC: displacement control.

\begin{tabular}{c|c|c}
\hline Test & $\begin{array}{c}\text { Penetration to } \\
w / D(-)\end{array}$ & $\begin{array}{c}\text { Dissipation } \\
\text { mode }\end{array}$ \\
\hline LC $-0.5 D$ & 0.5 & LC \\
DC $-0.5 D$ & 0.5 & DC \\
LC $-1 D$ & 1.0 & LC \\
DC $-1 D$ & 1.0 & DC \\
\hline
\end{tabular}

The model PPP was $50 \mathrm{~mm}$ in diameter and manufactured from aluminium with a polished surface (see Figure 1). All tests were performed at an acceleration of $10 \mathrm{~g}$, representing an equivalent field scale PPP with a diameter of $500 \mathrm{~mm}$. The probe was instrumented with a $100 \mathrm{~N}$ S-type load cell designed in-house to measure the penetration resistance and $50 \mathrm{kPa}$ range pore pressure transducers located at the invert and midface positions.

Commercial kaolin clay was preconsolidated in-flight at a centrifuge acceleration of $30 \mathrm{~g}$, before reducing the acceleration to $10 \mathrm{~g}$ for testing. This resulted in a constant overconsolidation ratio $(O C R)$ of 3 and an elevated strength gradient which represents surficial soil conditions commonly found offshore. Penetration was conducted at a rate $(0.125 \mathrm{~mm} / \mathrm{s}$ at model scale), which was sufficiently fast to avoid any significant dissipation of excess pore pressures during the embedment of the PPP. At the end of penetration dissipation was allowed until the penetration induced excess pore pressures had reduced to $\sim 10 \%$ of their initial value. To characterise the sample and benchmark the penetration resistance measurements, miniature T-bar penetrometer tests were conducted to measure the undrained strength profile of the sample. 
Schneider, M. A., Stanier, S. A., Chatterjee, S., White, D. J. and Randolph, M. F.

The parkable piezoprobe for determining $c_{v}$ and strength - modelling and interpretation methods

\section{NUMERICAL MODELLING (LDFE)}

To correctly capture the soil heave around the PPP during undrained penetration, coupled large deformation FE-analyses (LDFE) were performed. An axisymmetric model based on the “Remeshing and Interpolation Technique with Small Strains (RITSS)” methodology of Hu \& Randolph (1998) was developed and implemented in ABAQUS (Dassault Systèmes, 2011), following Wang et al. (2010). In brief, each large deformation analysis is subdivided into many consecutive small strain simulation steps. To preclude severe mesh distortions the whole soil domain is remeshed at the beginning of each step, with all stresses and state parameters remapped from the old mesh of the previous step, to the new undistorted mesh, using the super-convergent patch recovery technique proposed by Zienkiewicz \& Zhu (1992). The consolidation phase was simulated using a final conventional small strain analysis, i.e. without further remeshing. The derived stresses and state parameters at the target penetration depth served as initial conditions for the dissipation stage.

The analysis domain was specified to be sufficiently large - with a depth of $10 \mathrm{D}$ and a radius of $8 D$ - as to eliminate boundary effects. The Modified Cam Clay soil model (MCC) of Roscoe and Burland (1968) was used in combination with a void ratio-dependent permeability function, developed by Mahmoodzadeh et al. (2014), calibrated to Rowe cell tests performed on kaolin clay. Free drainage was allowed at the mudline but precluded where the soil was in contact with the probe. This was achieved by prescribing hydrostatic conditions (pore pressure relative to the vertical location of each node on the free surface) at all freely draining surface boundary nodes. The PPP was modelled as a rigid body with a smooth interface to represent the polished metal surface of the centrifuge model.

The penetration process was modelled fast enough for drainage to be negligible, with dimensionless velocity $V\left(=v D / c_{v}\right)$ equal to 100 at the invert of the device at the start of the penetration process. Additionally, a minimum time increment was enforced to avoid spurious 
Schneider, M. A., Stanier, S. A., Chatterjee, S., White, D. J. and Randolph, M. F.

The parkable piezoprobe for determining $c_{v}$ and strength - modelling and interpretation methods

non-physical oscillations in the pore pressure fields (Vermeer \& Verruijt, 1981), following the guidance given in the ABAQUS user manual (Dassault Systèmes, 2011).

The numerical procedures were initially checked and benchmarked against existing solutions published by Chatterjee et al. (2014) and Stanier \& White (2014). Additionally, the validity of the modelling assumptions (mesh density, penetration speed, surface drainage assumption and permeability function) was assessed via sensitivity analyses that are summarised in the Appendix.

\section{COMPARISON OF RESULTS: CENTRIFUGE VS. LDFE}

For the following comparisons all units are given in prototype scale using the applicable scaling laws according to Garnier et al. (2007). For the numerical back-analyses a probe diameter of $0.5 \mathrm{~m}$ was modelled (prototype scale). All penetration phases simulated a target penetration depth of either $0.5 \mathrm{D}$ or $1 \mathrm{D}$. Only the load-controlled (LC) dissipation phases that followed were modelled, since these tests are more representative of the anticipated field use of the device (i.e. dissipation under the self-weight of the device). The simulation parameters and in-situ stress state are summarised in Table 2.

Table 2: Modified Cam Clay simulation parameters (after Stewart, 1992)

\begin{tabular}{l|c}
\hline Parameter & Value \\
\hline Critical state constant, $M$ & 0.92 \\
Void ratio on CSL (at $\left.\mathrm{p}^{\prime}=1 \mathrm{kPa}\right), e_{c s}$ & 2.14 \\
Slope of normal consolidation line, $\lambda$ & 0.205 \\
Slope of swelling line, $\kappa$ & 0.044 \\
Poisson's ratio, $v$ & 0.3 \\
Effective unit weight, $\gamma^{\prime}\left(\mathrm{kN} / \mathrm{m}^{3}\right)$ & 6 \\
Unit weight of water, $\gamma_{w}\left(\mathrm{kN} / \mathrm{m}^{3}\right)$ & 10 \\
\hline Modelled in-situ stress state & Value \\
\hline Over-consolidation ratio, OCR & 3 \\
\hline
\end{tabular}


For simplicity in back-analysing the centrifuge experiments, isotropic permeability $\left(k_{h}=k_{v}\right)$ was assumed. The influence of potential anisotropic permeability is discussed later in the paper. A small surcharge of $0.25 \mathrm{kPa}$ was applied at the mudline to improve numerical stability and create a non-zero strength intercept at the soil surface, $s_{u, m}$ consistent with the centrifuge sample, which had been lightly scraped to flatten the sample surface prior to testing.

\section{Sample properties}

Figure 3 shows the measured effective unit weight and undrained shear strength profiles of the centrifuge soil sample. Undrained strength measurements are presented with and without shallow depth corrections (White et al., 2010), which in this case have minimal influence on the interpretation due to the lightly over-consolidated state of the sample. A T-bar factor of 10.5 was adopted, corresponding to an intermediate T-bar roughness (Randolph \& Houlsby, 1984). The MCC model formulation inbuilt in ABAQUS has a circular yield surface and plastic potential in the $\pi$-plane. The corresponding undrained strength profile in the numerical simulations, which as shown in Figure 3b, is in close agreement with the measured strength in the centrifuge sample, was derived from (Wood, 1990):

$$
\begin{gathered}
s_{u}=\frac{M}{2}\left(\frac{n_{p}}{r}\right)^{\Lambda} \frac{\left(1+2 K_{0, o c}\right)}{3} \sigma_{v}^{\prime} \quad n_{p}=\frac{\left(1+2 K_{0, n c}\right)}{\left(1+2 K_{0, o c}\right)} O C R \quad r=\exp \left(\frac{M-\eta}{M}\right) \\
K_{0, n c}=1-\sin \varphi^{\prime} \quad K_{0, o c}=K_{0, n c} O C R^{(\sin \varphi)}
\end{gathered}
$$

with the parameters $\eta$ and $\Lambda$ defined as $q / p^{\prime}$ and $(\lambda-\kappa) / \lambda$, respectively. 
The parkable piezoprobe for determining $c_{v}$ and strength - modelling and interpretation methods

\section{Penetration stage}

The total vertical resistance $V_{\text {tot }}$ during PPP penetration is the sum of the geotechnical resistance of the soil $V_{\text {geot }}$ and the soil buoyancy $V_{b}\left(=f_{b} \gamma^{\prime} v_{\text {sub }}\right)$ where $f_{b}$ is an adjustment factor and $v_{\text {sub }}$ the embedded volume. The bearing capacity equation, rearranged in terms of the bearing capacity factor or normalised penetration resistance $N_{c, \text { nom }}$, is therefore:

$$
N_{c, \text { nom }}=\left(\frac{V_{\text {geot }}}{A_{\text {nom }} s_{u 0}}\right)=\left(\frac{V_{\text {tot }}}{A_{\text {nom }} S_{u 0}}\right)-f_{b}\left(\frac{\gamma^{\prime} V_{\text {sub }}}{A_{\text {nom }} s_{u 0}}\right) \quad \text { with } \quad A_{\text {nom }}=\frac{\pi D^{2}}{4}
$$

The initial undrained shear strength at the corresponding invert depth is represented by $s_{u} 0$ and can be calculated using Equation 1. The contribution of soil buoyancy in excess of Archimedes' principle, caused by the generation of soil heave, is accounted for by the factor $f_{b}$. Stanier \& White (2014) determined that the $f_{b}$ factor for a hemiball penetrometer, which has exactly the same geometry as the parkable piezoprobe, could be adequately captured by

$$
f_{b, P P P} \cong f_{b, \text { hemi }}=1.19+0.06\left(k_{s u} D / s_{u, a v g}\right) \quad \text { with } \quad s_{u, a v g}=s_{u, m}+0.5 k_{s u} D
$$

where $s_{u, m}$ represents the soil strength at the mudline, $k_{s u}$ the strength gradient with depth and $D$ the probe diameter. For a given embedment depth, $w$, the embedded volume $v_{\text {sub }}$ of the device (below the original soil surface) can be determined by

$$
\begin{gathered}
v_{\text {sub }}=\frac{\pi w^{2}}{3}(1.5 D-w) \quad \text { for } \quad w \leq 0.5 D \\
v_{\text {sub }}=\frac{\pi D^{3}}{12}+\frac{\pi D^{2}}{8}(2 w-D) \quad \text { for } \quad 0.5 D<w \leq D
\end{gathered}
$$

The linear shear strength profile (Figure 3b) is used to estimate the penetration resistance for the PPP through: (i) direct numerical modelling of the experiments and via (ii) a prediction of the undrained penetration resistance using the model for undrained hemiball penetration resistance based on the equations above and the functions for $N_{c, \text { nom }}$ given by Stanier \& White 
(2014). Figure 4 compares the numerical simulations, centrifuge measurements and calculation model given above. All four centrifuge tests are in relatively close agreement with each other, showing excellent test repeatability and lateral homogeneity of the centrifuge sample. There is also very good agreement between the experimental results and the numerical simulations. The bearing capacity model after Stanier \& White (2014), which was originally derived from parametric large deformation FE-analyses based on the Tresca model, provides upper and lower bounds that fall on either side of the MCC simulation. The normalised resistance of the smooth Tresca simulation based model fit is $~ 15 \%$ less than the MCC simulations. This discrepancy is not unexpected and is partly due to the different yield surface shapes assumed by the two models (MCC: circular vs. Tresca: hexagonal). The difference is close to the maximum deviation theoretically possible between the two models $(2 / \sqrt{ } 3 \sim 15.5 \%$ if plane strain shear conditions are prevalent) and may therefore also be a consequence of slight drainage during the penetration phase. Similar observations were made by Mahmoodzadeh et al. (2015) where coupled MCC penetrometer analyses yielded between 9 and 12\% higher penetration resistance compared to equivalent Tresca simulations.

The simulations illustrate that the prediction model of Stanier \& White (2014) is adequate to either: (i) deduce an undrained strength profile at shallow depth if the vertical soil resistance is measured (as in this instance), (ii) deduce a spot measurement of undrained strength if the penetration depth and self-weight of a PPP are known; or to (iii) estimate the self-weight requirements of the PPP for different anticipated soil conditions.

\section{Dissipation stage}

A challenge associated with developing a simple interpretation method from numerical simulations using the Modified Cam Clay soil model, is to calculate an appropriate value for the consolidation coefficient with which to normalise the simulated dissipation response. This is a necessary step in order to determine the non-dimensional time required for $50 \%$ 
dissipation to occur $\left(T_{50}\right)$ for general application of the interpretation method. The choice is a compromise, as in reality, dissipation is a highly non-linear process with spatial and temporal variations in stiffness, so a representative value for $c_{v}$ is required, and must be selected carefully. Depending on the current soil state (e.g. lightly over-consolidated or normally consolidated conditions) the operational stiffness can change during the course of the dissipation stage (i.e. transitioning between the reloading and normal compression lines). The initial coefficient of consolidation $c_{v 0}$ is defined conventionally (e.g. Chatterjee et al. (2014); Chatterjee et al. (2013)) as a function of the slope of the normal compression line, $\lambda$. The implications of this assumption will be revisited later in the paper.

The initial coefficient of consolidation $c_{v 0}$ at the invert depth, corresponding to the start of the dissipation phase, may be estimated as

$$
C_{v 0}=\frac{k_{0}}{m_{v} \gamma_{w}}=\frac{k_{0}\left(1+e_{0}\right) p_{0}^{\prime}}{\lambda \gamma_{w}}
$$

The parameter is defined as a function of the initial permeability $k_{0}$, void ratio $e_{0}$ and mean effective stress $p_{0}^{\prime}$ at the same embedment. Figure 5 presents dimensional and nondimensional interpretations of the centrifuge measurements alongside their numerical simulations for a normalised embedment of $w / D=0.5$ and $w / D=1$. The centrifuge measurements are shown with and without the correction suggested by Sully et al. (1999), where back-extrapolation is used to estimate the initial excess pore pressure at the start of dissipation (the so-called root-time method). This correction, which was originally suggested for non-monotonic dissipation measurements, is necessary to account for lag in the measured pore pressure response and to ensure that an appropriate initial excess pore pressure is used in the following normalisation. The excess pore pressure, $\Delta u(t)$, is normalised by its initial value, $\Delta u_{i}$, at the onset of the dissipation stage, whilst the normalised time, $T$, is expressed as a function of the dissipation time $t$ as: 
Schneider, M. A., Stanier, S. A., Chatterjee, S., White, D. J. and Randolph, M. F.

The parkable piezoprobe for determining $c_{v}$ and strength - modelling and interpretation methods

$$
T=\frac{C_{v 0} t}{D^{2}}
$$

The normalised dissipation responses are of similar shape for both the numerical LDFEsimulations and the centrifuge tests. In this interpretation the non-dimensional dissipation curves extracted from the numerical simulations at the invert and midface locations are normalised by the same value of $c_{v 0}$, taken at the invert depth. Therefore, the coefficient of consolidation, $c_{v 0}$, derived by matching the experimental and numerical dissipation curves (varying the $c_{v 0}$ value used to normalise the experimental measurements), should be very similar for both the midface and invert locations.

Table 3: Summary of $c_{v 0}$ and $T_{50}$ from experiments and simulations

\begin{tabular}{c|c|c|c|c}
\hline \multirow{2}{*}{$w / D$} & \multicolumn{2}{|c|}{$c_{v 0}\left(\mathrm{~m}^{2} / \mathrm{yr}\right)$} & \multicolumn{2}{c}{$T_{50}(-)$} \\
\cline { 2 - 5 } & $\begin{array}{c}\text { inferred } c_{v 0} \\
\text { (back-fitting to LDFE) }\end{array}$ & $\begin{array}{c}\text { simulated } c_{v 0} \\
\text { (Equation 5) }\end{array}$ & invert & midface \\
\hline 0.5 & 0.6 & 0.6 & 0.005 & 0.007 \\
1.0 & 2.4 & 0.67 & 0.009 & 0.010 \\
\hline
\end{tabular}

Table 3 compares the inferred coefficients of consolidation with the simulated values. Additionally, the normalised times $T_{50}$ required to dissipate $50 \%$ of the accumulated excess pore pressures, are presented in the table. For an embedment depth of $0.5 D$, the dissipation response is well-predicted by the LDFE-simulation for both the invert and midface locations. As a consequence, excellent agreement between the inferred (back-fitted by varying the value of $c_{v 0}$ used in the normalisation until the experimental and numerical curves aligned) and the simulated $c_{v 0}$ values is also achieved in non-dimensional terms. For an embedment depth of $1 D$ and the input model parameters adopted, the experimentally measured dissipation is significantly faster than that simulated. The back-fitted coefficient of consolidation is consequently higher than the value prescribed in the numerical simulations. 
There are a number of potential causes for this disparity. For example, there is some suggestion in the literature that the horizontal permeability, $k_{h}$, of kaolin clay is greater than the vertical permeability, $k_{v}$, by a factor of 2-3 (Al-Tabbaa \& Wood, 1987). Under the assumption that radial dissipation dominates and is therefore mostly controlled by the horizontal permeability, the back-calculated coefficient of consolidation, $c_{v 0}$, could increase by a factor of about $n_{k}=k_{h} / k_{v} \sim 3$.

The magnitude of this discrepancy when comparing the LDFE and centrifuge results at the two embedment depths prompted a further parametric study varying the parameters that control the consolidation coefficient to systematically identify the most likely source. This study provides a better basis to interpret PPP dissipations in a given soil sample, beyond a single estimate of $c_{v 0}$.

\section{PARAMETRIC STUDY AND INTERPRETATION MODEL}

\section{Procedure and assumptions}

The parametric study examines the effect of permeability anisotropy, over-consolidation ratio and also changes in the elastic stiffness. The operative coefficient of consolidation, $c_{h 0}$, is explored by varying these parameters systematically. The results are interpreted by adjusting the previously-defined coefficient of consolidation, $c_{v 0}$, by factors $f_{k}$, and $f_{s t}$ which account for the effect of anisotropic permeability and the operative stiffness, respectively, resulting in:

$$
c_{h 0}=f_{k} f_{s t} c_{v 0} \quad \text { with } \quad c_{v 0}=\frac{k_{v}\left(1+e_{0}\right) p_{0}^{\prime}}{\lambda \gamma_{w}}
$$

The $c_{v 0}$ value adopted in the previous section corresponds to a value measured in an oedometer or conventional Rowe cell test, where vertical pore water flow typically dominates. For soils with anisotropic permeability, the isotropic permeability, $k_{0}$, in Equation 5, is replaced by the vertical permeability, $k_{v}$, in the definition of $c_{v 0}$. A dimensionless time, $T^{*}$, is then defined in 
The parkable piezoprobe for determining $c_{v}$ and strength - modelling and interpretation methods

terms of the operative coefficient of consolidation, $c_{h 0}$, and two further influence factors, $f_{R I}$ and $f_{w}$, which account for the influence of the rigidity index and the embedment depth, to give:

$$
T^{*}=f_{R I} f_{w} \frac{c_{h 0} t}{D^{2}}
$$

Suitable functions to generate these factors have been derived from the LDFE-simulations summarised in Table 4. The rigidity index in the MCC Model was adjusted by varying the Poisson's ratio.

Table 4: Summary of scope of parametric analyses (bold text: baseline case)

\begin{tabular}{l|c|c|c}
\hline Parameter & Value & Target Sensitivity & Target Factor \\
\hline Poisson's ratio, $v$ & $0.25 / \mathbf{0 . 3 0} / 0.35$ & Rigidity index $I_{R}$ & $f_{R I}$ \\
Permeability ratio, $n_{k}=k_{h} / k_{v}$ & $\mathbf{1} / 2 / 3$ & Anisotropy: $k_{h}>k_{v}$ & $f_{k}$ \\
Slope of normal consolidation line, $\lambda$ & $0.110 / \mathbf{0 . 2 0 5} / 0.352$ & Ratio of $\kappa / \lambda *$ & $f_{s t}$ \\
Slope of swelling line, $\kappa$ & $\mathbf{0 . 0 4 4} / 0.062 / 0.082$ & Ratio of $\kappa / \lambda *$ & $f_{s t}$ \\
Over-consolidation ratio, $O C R$ & $\mathbf{1} / 1.5 / 2 / 3 / 4 / 5$ & Effect of $O C R$ & $f_{s t}$ \\
Normalised embedment depth, $w / D$ & $0.3 / 0.4 / \mathbf{0 . 5} \ldots 1$ & Effect of embedment & $f_{w}$ \\
\hline
\end{tabular}

$*$ The $\lambda$ and $\kappa$ values selected lead to ratios of $\kappa / \lambda=0.125,0.215,0.3,0.40$.

For each simulation one parameter was varied from the baseline case (values given in bold in Table 4) to assess its influence on the non-dimensional dissipation response. For all parameter combinations (14 in total) an initial undrained penetration to a depth of $1 D$ was simulated (at a dimensionless penetration rate, $V=100$ ). A smooth probe with a diameter of $0.25 \mathrm{~m}$ was considered following Chatterjee et al. (2014). An effective unit weight of $5 \mathrm{kN} / \mathrm{m}^{3}$ was adopted for the soil and a constant vertical permeability of $k_{v}=10^{-9} \mathrm{~m} / \mathrm{s}$ was assumed for all cases. To improve numerical stability and generate a small strength intercept at the soil surface a surcharge of $1 \mathrm{kPa}$ was introduced at the mudline (following Chatterjee et al., 2014). All other parameters remain as in the previous section (see Table 2). 
Consolidation phases corresponding to normalised embedment depths of $w / D=0.3,0.4,0.5 \ldots$

1 were analysed. Depths less than $0.3 D$ were not modelled as this is approximately the minimum depth required for both the midface and invert pore pressure measurement locations to be sufficiently embedded to achieve reliable dissipation measurements for the current configuration of device. Figures that illustrate the influence of the parameters and the effectiveness of the proposed factors in collapsing all dissipation responses to a single curve are given in the following sections. For brevity, only dissipation at the invert location for an embedment of $0.5 D$ is shown, however the midface performance and the responses at all other embedment depths were very similar.

\section{Effect of rigidity index}

To investigate the influence of the rigidity index $I_{R}=G / s_{u}$ on the dissipation solution, LDFEcalculations assuming different values of Poisson's ratio were conducted. The rigidity index is estimated based on the initial undrained shear strength $s_{u 0}$ and shear modulus $G_{0}$ at the invert level of the probe. The initial shear stiffness $G_{0}$ can be calculated as:

$$
G_{0}=\frac{3 p_{0}^{\prime}\left(1+e_{0}\right)(1-2 v)}{2 \kappa(1+v)}
$$

Poisson's ratios of $0.25,0.30$ and 0.35 correspond to rigidity indexes of 130,100 and 72 , respectively. For the cone and ball penetrometers a function for the influence factor due to changes in rigidity index, referred to as $f_{R I}$ here, is typically defined as (Teh \& Houlsby, 1991):

$$
f_{R I}=\frac{1}{\left(I_{R}\right)^{\beta}}
$$

The numerical simulations with rigidity indexes varying in the range of 72-130 indicated that the appropriate value for $\beta$ for the PPP was objectively defined via best fitting as 0.001 . This 
Schneider, M. A., Stanier, S. A., Chatterjee, S., White, D. J. and Randolph, M. F.

The parkable piezoprobe for determining $c_{v}$ and strength - modelling and interpretation methods

results in an insignificant influence factor $f_{R I}$ of effectively unity. In other words, the rigidity index has practically no influence on the dissipation response of the PPP due to the lack of confinement at shallow embedment; therefore the influence factor $f_{R I}$ can be discarded. This is in contrast to cone and ball penetrometers, where values of 0.5 (Mahmoodzadeh et al., 2014) and 0.25 (Mahmoodzadeh et al., 2015) apply, respectively.

\section{Effect of permeability anisotropy}

The permeability ratio $n_{k}$ was varied to evaluate the effect of anisotropic flow conditions. A function for the influence factor $f_{k}$ is defined as follows:

$$
f_{k}=\frac{2 n_{k}+1}{3}
$$

Figure 6 illustrates that this function collapses all responses to a unique curve, which means that the operative permeability is essentially the geometric mean of the permeability components in three dimensions. An expression, yielding similar $f_{k}$ factors, was found by Wang et al. (2015) for the piezoball, with the slight deviation mainly due to the difference in device geometry and embedment conditions.

\section{Effect of operative stiffness for normally-consolidated conditions}

The influence of the operative stiffness was examined by changing the value of $\lambda$ or $\kappa$. The dissipation response normalized by $c_{v 0}$ varies with the ratio of $\kappa / \lambda$, and this is confirmed in Figure 7a, where two different parameter combinations - both with a ratio of $\kappa / \lambda$ of 0.4 - lead to the same dissipation response. For normally consolidated clay the influence factor, $f_{s t}$, can 
be defined as follows (Mahmoodzadeh et al., 2014 \& 2015), where $\alpha$ controls the influence of the stiffness ratio, $\kappa / \lambda$ :

$$
f_{s t}=\left(\frac{\lambda}{\kappa}\right)^{\alpha}
$$

A value of $\alpha \sim 0.25$ collapses all of the dissipation responses for normally consolidated soil, where the slope of the normal compression line dominates the operative stiffness, as shown in Figure 7b. Numerical analyses of piezocone dissipation tests by Mahmoodzadeh et al. (2014) revealed the same for the cone, namely that the stress-paths observed in the vicinity of the cone are partially influenced by the reloading stiffness, even for normally consolidated conditions. The responses for $\kappa / \lambda$ of 0.3 and 0.4 were almost indistinguishable, hence for clarity, the dissipation response for the $\kappa / \lambda$ equal to 0.3 case is omitted in Figure 7 a.

\section{Effect of over-consolidation ratio}

To calibrate the sensitivity of the dissipation response to lightly over-consolidated conditions, additional LDFE-simulations were run with constant OCR in the range of 1 to 5 .

Figure 8a illustrates the sensitivity of the dissipation response to these lightly overconsolidated conditions. The dissipation responses can be collapsed to a unique curve as shown in

Figure $8 \mathrm{~b}$ by modifying the stiffness factor for normally consolidated conditions, $f_{s t}$, by: (i) applying a factor $O C R^{4}$ to account for the difference in mean stress caused by overconsolidation (Mahmoodzadeh et al., 2014); and (ii) varying the weighting factor $\alpha$ in Equation 12, as a function of the OCR. The factor $f_{\text {st }}$ becomes

$$
f_{s t}=\left(\frac{\lambda}{\kappa}\right)^{\alpha} O C R^{\Lambda} \quad \text { with } \quad \Lambda=\frac{\lambda-\kappa}{\lambda}
$$


A suitable relationship for $\alpha$ was determined via back-fitting to the optimal values for each simulation (see Figure 9), resulting in

$$
\alpha=a \cdot e^{(b / O C R)} \quad \text { with } \quad a=0.647 \& b=-0.913
$$

This expression retains $\alpha=0.25$ at $O C R=1$ as determined previously. The change in $\alpha$ from $\sim 0.25$ for normally consolidated conditions to $\sim 0.55$ for an $O C R$ equal to 6 (where Equation 14 is approaching an asymptotic state) reflects that the reloading stiffness $\kappa$ increasingly dominates the dissipation response as the soil becomes more over-consolidated.

\section{Influence of embedment depth}

Finally, all dissipation responses for embedment depths in the range of 0.3 to $1 D$ can also be collapsed to a unique curve by applying a scaling factor that varies with embedment. This is useful for assessing the uncertainty in consolidation coefficient that would exist if the embedment of the PPP was not known.

For each analysis the fitting parameters $T_{50} *$ and $m *$, which describe the dissipation solution according to Equation 15, were back-fitted for all cases simulated, after accounting for the influence factors $f_{k}, f_{s t}$, and $f_{O C R}$.

$$
\frac{\Delta u}{\Delta u_{i}}=\frac{1}{1+\left(T / T_{50}\right)^{m}}
$$

The data points (finite element simulation increments) that make up the numerical dissipation responses generally had non-uniform time intervals. Consequently each data point was weighted in the back-fitting process so that its influence on the fitting process was proportional to the time interval that it represented. Figure 10 shows the dependency of the back-fitted parameters $T_{50} *$ and $m^{*}$ on the normalised penetration depth $w / D$ for the invert location. Using the estimated average values of $T_{50}{ }^{*}$ a suitable relationship for the influence 
factor $f_{w}$ was established, collapsing all of the dissipation responses to a unique curve equal to that for an embedment of $0.5 D$, given by:

$$
f_{w}=\frac{\overline{T_{50}^{*}}(w / D=0.5)}{\overline{T_{50}^{*}}(w / D)} \quad \leftrightarrow \quad f_{w}=a(w / D)^{b} \quad \text { with } \quad a=0.65 \& b=-0.67
$$

Figure 11 shows the fit of Equation 16 versus the optimally back-fitted values for $w / D$ in the range of 0.3 to 1 . For simplicity a single relationship for $f_{w}$ is recommended, which is valid for both the invert and the midface pore pressure sensor locations, with minimal error.

The operative coefficient of consolidation $c_{h o}$ can be determined with reasonable accuracy even if the exact embedment depth of the probe is unknown (i.e. the embedment is not measured). In this event an $f_{w}$ factor of 1 - corresponding to a normalised embedment, $w / D$, of 0.5 - can be assumed, resulting in a maximal deviation from the optimal value of $\sim 50 \%$ for an actual embedment of $0.3 D$ or $\sim 35 \%$ for an embedment of $1 D$, assuming the probe embedment is within this range.

\section{Proposed interpretation model}

Using the influence factor relationships developed in the preceding sections, unique dissipation curves for the invert and midface locations can be determined for a normalised embedment, $w / D$, of 0.5 , which can then be used to back-analyse any field dissipation tests with reasonable accuracy. Values for $T_{50}{ }^{*}$ and $\mathrm{m}^{*}$ are given in Table 5 for general use for both the midface and invert pore pressure sensor locations.

Table 5: Dissipation solution fitting parameters for the proposed interpretation model.

\begin{tabular}{c|c|c}
\hline Location & $T_{50} *(-)$ & $m^{*}(-)$ \\
\hline Invert & 0.035 & 1.05 \\
Midface & 0.041 & 1.05 \\
\hline
\end{tabular}


Field data from parkable piezoprobe dissipation tests can be interpreted with the following simple procedure:

1. Evaluate $f_{w}$ (Equation 16) if embedment was measured; otherwise assume an influence factor $f_{w}$ of 1 .

2. Normalise the dissipation data according to Equations 8 (note: $f_{R I}=1$ ) and fit the resulting data to the curve described by Equation 15 (using the appropriate parameters from Table 5) by varying the operative coefficient of consolidation, $c_{h o}$, used in the normalisation.

3. Optional: Estimate the sensitivity of the oedometric coefficient of consolidation, $c_{v 0}$, using Equations 7, 11, 13 and 14 and an expected potential range for the various unknown soil parameters.

\section{Verification of interpretation model}

The interpretation model was verified by performing additional LDFE simulations for randomly selected embedment depths and parameter combinations not used in the development of the interpretation model, but within the bounds of the parametric study presented in the previous section. In Figure 12 the operative coefficient of consolidation, $c_{h o}$, inferred from the interpretation model is plotted against the expected value determined from Equation 7 for fifteen validation cases. The interpretation model proves to be accurate and robust for all cases studied with errors generally within $\pm 10 \%$.

\section{EXAMPLE APPLICATION}

The proposed interpretation model was next used to reinterpret the centrifuge test results, following the steps developed in the previous section: 
Schneider, M. A., Stanier, S. A., Chatterjee, S., White, D. J. and Randolph, M. F.

The parkable piezoprobe for determining $c_{v}$ and strength - modelling and interpretation methods

1. Appropriate values of the embedment influence factor, $f_{w}$, were taken for the embedment depths of $0.5 D$ and $1 D$.

2. The measured dissipation data was then normalised according to Equation 8, (taking $f_{R I}=1$ ). Equation 15 was used to generate a model fit (using the appropriate best estimate parameters for the test data as summarised in Table 5) to which the measured dissipation was back-fitted by adjusting the operative coefficient of consolidation, $c_{h o}$. This procedure yielded best estimate values of $c_{h 0}$ of $3.1 \mathrm{~m}^{2} / \mathrm{yr}$ and $14 \mathrm{~m}^{2} / \mathrm{yr}$ for embedment depths of $0.5 D$ and $1 D$, respectively

3. The sensitivity of the oedometric coefficient of consolidation, $c_{v 0}$, was estimated over an expected potential range for the various unknown soil parameters via a Monte Carlo analysis using the parameters outlined in Table 6.

Table 6: Soil parameter inputs to Monte Carlo analysis.

\begin{tabular}{c|c|c|c|c}
\hline Input parameter & Lower bound & Mean value, $\mu$ & Upper bound & Distribution \\
\hline$\kappa / \lambda$ & 0.125 & 0.215 & 0.4 & $*$ \\
$n_{k}$ & 1 & 2 & 3 & uniform \\
OCR & $N / A$ & 3 & $N / A$ & N/A \\
\hline
\end{tabular}

* Normal distributions for $\lambda$ and $\kappa$, with $\mu_{\lambda}=0.205$ and $\mu_{\kappa}=0.044$ (after Stewart, 1992) and corresponding standard deviations $\sigma_{\lambda}$ and $\sigma_{\kappa}$ chosen so that $\sim 99 \%$ of all resulting $\kappa / \lambda$-ratios fall within stated range

For comparison purposes the best estimate $c_{h 0}$ values derived from step 2, valid for an $O C R$ of 3 , were converted to values representative of normally consolidated conditions, using Equation 13 ( $\lambda$ and $\kappa$ assumed according to Table 2). The estimated $c_{h 0, n c}$ values are in good agreement with trends back-extrapolated from other published data for UWA kaolin clay under higher stress conditions (Figure 13). The interpretation of the operative coefficient of consolidation at the invert, $c_{h 0}$, was consistent irrespective of the considered pore pressure sensor location (midface or invert), demonstrating that the interpretation model derived is internally consistent. 
Schneider, M. A., Stanier, S. A., Chatterjee, S., White, D. J. and Randolph, M. F.

The parkable piezoprobe for determining $c_{v}$ and strength - modelling and interpretation methods

The estimated distributions of the oedometric $c_{v 0}$ values, derived via the Monte Carlo simulation conducted as part of step 3, are presented in Figure 14 . The $5 \%$ and $95 \%$ percentiles, which are often used in design, are also indicated in the figure. This latter example serves as a demonstration of how the PPP interpretation method developed can be used to interpret dissipation data to estimate operative coefficients of consolidation, $c_{h 0}$, and a potential range of equivalent oedometric coefficient of consolidation, $c_{v 0}$, given approximate expected ranges of the governing soil parameters. The range of $c_{v 0}$ can be refined using other site investigation data as input as it becomes available.

\section{CONCLUSIONS}

The parkable piezoprobe is a promising tool for economically measuring the coefficient of consolidation offshore. This paper has presented results from centrifuge model tests and a comprehensive suite of large deformation finite element simulations investigating the performance of the parkable piezoprobe have been reported, leading to the following conclusions:

- All centrifuge measurements of the undrained penetration stage were in excellent agreement with the results of the numerical large deformation simulations. The experimentally recorded and numerically simulated dissipation responses were consistent with each other in both non-dimensional and dimensional terms.

- Back-analyses of the centrifuge tests revealed that the model of Stanier \& White (2014) is adequate to define the effective weight of device required to achieve a penetration in the range of $0.3-1 D$ for anticipated in-situ conditions.

- A simple interpretation model for inferring the operative coefficient of consolidation $c_{h o}$ from field test data was developed from a series of LDFE analyses. In its simplest form - with no prior knowledge of soil properties or the initial embedment depth of the device - reasonably accurate estimates of the operative coefficient of 
Schneider, M. A., Stanier, S. A., Chatterjee, S., White, D. J. and Randolph, M. F.

The parkable piezoprobe for determining $c_{v}$ and strength - modelling and interpretation methods

consolidation, $c_{h o}$, can be achieved. More accurate estimates can be achieved in the event that some of the soil parameters are measured independently.

- Due to the radial drainage of excess pore pressures around the device dissipation tests for the PPP $(D=250 \mathrm{~mm})$ are about twice as fast as for the SMARTPIPE ( $D=225 \mathrm{~mm}$ ), where planar drainage dominates. Piggybacking these tests onto a site investigation survey would therefore provide an economical way to reliably gain near surface seabed properties.

\section{ACKNOWLEDEMENTS}

The research presented in this paper is part of the activities of the Centre for Offshore Foundation Systems (COFS), currently supported as a node of the Australian Research Council Centre of Excellence for Geotechnical Science and Engineering. The second author is supported by an ARC DECRA Fellowship DE170100119. This work has been further supported by Shell, via the Shell EMI Chair in Offshore Engineering held by the fourth author, and by Fugro via the Fugro Chair in Geotechnics held by the fifth author. The first author is grateful for the support provided by an International Postgraduate Research Scholarship (IPRS) from the Australian Government. 
Schneider, M. A., Stanier, S. A., Chatterjee, S., White, D. J. and Randolph, M. F.

The parkable piezoprobe for determining $c_{v}$ and strength - modelling and interpretation methods

\section{NOTATION}

$A_{\text {nom }} \quad$ nominal area

$a, b \quad$ fitting parameters

$c_{h} \quad$ operative coefficient of consolidation

$c_{h o} \quad$ initial operative coefficient of consolidation at invert level

$c_{h 0, n c} \quad$ initial $c_{h o}$ at invert level (converted to normally consolidated conditions)

$c_{v} \quad$ coefficient of consolidation

$C_{v 0} \quad$ initial coefficient of consolidation at invert level

$D \quad$ device diameter

$e_{0} \quad$ initial void ratio

$e_{c s} \quad$ void ratio on critical state line at $p^{\prime}=1 \mathrm{kPa}$

$f_{b} \quad$ buoyancy factor

$f_{k} \quad$ influence factor for anisotropic flow conditions

focr influence factor for over-consolidation ratio

$f_{R I} \quad$ influence factor for rigidity index

$f_{s t} \quad$ influence factor for operative stiffness

$f_{w} \quad$ influence factor for embedment depth

G shear modulus

$G_{0} \quad$ initial shear modulus

$I_{R} \quad$ rigidity index

$K_{0, n c} \quad$ earth pressure coefficient at rest (NC-soil)

$K_{0, o c} \quad$ earth pressure coefficient at rest (OC-soil)

$k_{0} \quad$ isotropic permeability

$k_{h} \quad$ horizontal permeability component

$k_{s u} \quad$ undrained shear strength gradient

$k_{v} \quad$ vertical permeability component

$M \quad$ slope of critical state line in $q$ - $p$ ' space

$m, m^{*} \quad$ fitting parameter for consolidation solution

$m_{v} \quad$ coefficient of volume compressibility

$N_{c, \text { nom }} \quad$ nominal bearing capacity factor

$N_{T} \quad$ bearing capacity factor for T-Bar

$n_{k} \quad$ permeability ratio

$n_{p} \quad$ isotropic over-consolidation ratio

OCR over-consolidation ratio

$p$ ' mean effective stress

$p_{0}$, initial mean effective stress

$q \quad$ deviator stress

$r \quad$ ratio of pressures on normal compression and critical state line

$s_{u} \quad$ undrained shear strength

$s_{u 0} \quad$ initial undrained shear strength

$s_{u, a v g} \quad$ average undrained shear strength

$s_{u, m} \quad$ undrained shear strength at mudline

$T$ dimensionless time

$T^{*} \quad$ adjusted dimensionless time

$T_{50}$ dimensionless time to reach $50 \%$ of dissipation

$T^{*}{ }_{50} \quad$ adjusted dimensionless time to reach $50 \%$ of dissipation 
Schneider, M. A., Stanier, S. A., Chatterjee, S., White, D. J. and Randolph, M. F.

The parkable piezoprobe for determining $c_{v}$ and strength - modelling and interpretation methods

$t \quad$ time

$t_{50} \quad$ time to reach $50 \%$ of dissipation

$V \quad$ dimensionless velocity

$V_{b} \quad$ soil buoyancy

$V_{\text {geot }} \quad$ geotechnical soil resistance

$V_{\text {tot }} \quad$ total soil resistance

$v_{\text {sub }} \quad$ submerged volume

w embedment depth

$\alpha \quad$ exponent regarding stiffness contributions

$\beta \quad$ exponent regarding influence of rigidity index

$\Delta u \quad$ excess pore water pressure

$\Delta u_{i} \quad$ initial excess pore water pressure

$\gamma$, effective unit weight

$\gamma_{\text {sat }} \quad$ saturated bulk unit weight

$\gamma_{w} \quad$ unit weight of water

$\eta \quad$ stress ratio $q / p$,

$\varphi$, internal friction angle

$\kappa \quad$ slope of swelling line

$\Lambda \quad$ plastic compression ratio

$\lambda$ slope of normal consolidation line

$v \quad$ Poisson ratio

$\sigma_{v}$, effective vertical stress 
Schneider, M. A., Stanier, S. A., Chatterjee, S., White, D. J. and Randolph, M. F.

The parkable piezoprobe for determining $c_{v}$ and strength - modelling and interpretation methods

\section{REFERENCES}

Al-Tabbaa, A. \& Wood, D. M. (1987). Some measurements of the permeability of kaolin. Géotechnique 37, No. 4, 499-503.

An, H., Weidong, Y., Cheng, L., Draper, S., Zhao, M., Tang, G., Zhang, Y. \& Hortin, P. (2016). Detecting local scour using contact image sensors. ASCE Journal of Hydraulic Engineering, available ahead of print online, doi: 10.1061 / (ASCE) HY.1943-7900.0001266.

Chatterjee, S., Randolph, M. F. \& White, D. J. (2014). A parkable piezoprobe for measuring $\mathrm{C}_{\mathrm{v}}$ at shallow depths for offshore design. Géotechnique 64, No. 1, 83-88.

Chatterjee, S., White, D. J. \& Randolph, M. F. (2013). Coupled consolidation analysis of pipe-soil interactions. Canadian Geotechnical Journal 50, No. 6, 609-619.

Chow, S. H., O'loughlin, C. D. \& Randolph, M. F. (2014). Soil strength estimation and pore pressure dissipation for free-fall piezocone in soft clay. Géotechnique 64, No. 10, 817-827.

Cocjin, M. J., Gourvenec, S. M., White, D. J. \& Randolph, M. F. (2014). Tolerably mobile subsea foundations-observations of performance. Géotechnique 64, No. 11, 895-909.

Colreavy, C., O’Loughlin, C.D. \& Randolph, M.F. (2015). Estimating consolidation parameters from field piezoball tests. Géotechnique, 66, No. 4, 333-343.

Dassault Systèmes. (2011). Abaqus analysis user’s manual. Simulia Corporation. Providence, RI, USA.

Garnier, J., Gaudin, C., Springman, S. M., Culligan, P. J., Goodings, D., Konig, D., Kutter, B., Phillips, R., Randolph, M. F. \& Thorel, L. (2007). Catalogue of scaling laws and similitude questions in geotechnical centrifuge modelling. International Journal of Physical Modelling in Geotechnics 7, No. 3, 1.

Gourvenec, S. \& White, D. (2010). Consolidation around seabed pipelines. In Proceedings of Offshore Technology Conference. Offshore Technology Conference. 
Schneider, M. A., Stanier, S. A., Chatterjee, S., White, D. J. and Randolph, M. F.

The parkable piezoprobe for determining $c_{v}$ and strength - modelling and interpretation methods

Hill, A.J. \& Jacob, H. (2008). OTC 19528: In-situ measurement of pipe-soil interaction in deep water. Offshore Technology Conference, 5-8 May 2008, Houston, Texas, USA.

Hu, Y. \& Randolph, M. F. (1998). A practical numerical approach for large deformation problems in soil. International Journal for Numerical and Analytical Methods in Geomechanics 22, No. 5, 327-350.

Lehane, B. M., O'loughlin, C. D., Gaudin, C. \& Randolph, M. F. (2009). Rate effects on penetrometer resistance in kaolin. Géotechnique 59, No. 1, 41-52.

Mahmoodzadeh, H., Randolph, M. F. \& Wang, D. (2014). Numerical simulation of piezocone dissipation test in clays. Géotechnique 64, No. 8, 657-666.

Mahmoodzadeh, H., Wang, D. \& Randolph, M. F. (2015). Interpretation of piezoball dissipation testing in clay. Géotechnique 65, No. 10, 831-842.

Randolph, M. F. \& Hope, S. (2004). Effect of cone velocity on cone resistance and excess pore pressures. In Proceedings of Proc., Int. Symp. on Engineering Practice and Performance of Soft Deposits. Yodagawa Kogisha Co., Ltd., pp. 147-152.

Randolph, M. F. \& Houlsby, G. T. (1984). The limiting pressure on a circular pile loaded laterally in cohesive soil. Géotechnique 34, No. 4, 613-623.

Richardson, M. (2007). Rowe cell test on kaolin clay, COFS internal report. Centre for Offshore Foundation Systems, UWA, Crawley, Western Australia.

Roscoe, K. H. \& Burland, J. B. (1968). On the generalized stress-strain behaviour of wet clay. In Engineering plasticity (eds J. Heyman and F. A. Leckie). Cambridge University Press, Cambridge, UK, pp. 535-609.

Stanier, S. A., Schneider, M. A., White, D. J. \& Randolph, M. F. (2015). Modelling and interpretation of the parkable piezoprobe (PPP). UWA Report GEO15776.

Stanier, S. A. \& White, D. J. (2014). Shallow penetrometer penetration resistance. Journal of Geotechnical and Geoenvironmental Engineering 141, No. 3, 04014117.

Stewart, D. P. (1992). Lateral loading of piled bridge abutments due to embankment construction. PhD thesis, University of Western Australia. 
Schneider, M. A., Stanier, S. A., Chatterjee, S., White, D. J. and Randolph, M. F.

The parkable piezoprobe for determining $c_{v}$ and strength - modelling and interpretation methods

Sully, J. P., Robertson, P. K., Campanella, R. G. \& Woeller, D. J. (1999). An approach to evaluation of field CPTU dissipation data in overconsolidated fine-grained soils. Canadian Geotechnical Journal 36, No. 2, 369-381.

Teh, C. I. \& Houlsby, G. T. (1991). An analytical study of the cone penetration test in clay. Géotechnique 41, No. 1, 17-34.

Vermeer, P. A. \& Verruijt, A. (1981). An accuracy condition for consolidation by finite elements. International Journal for Numerical and Analytical Methods in Geomechanics 5, No. 1, 1-14.

Wang, D., White, D. J. \& Randolph, M. F. (2010). Large-deformation finite element analysis of pipe penetration and large-amplitude lateral displacement. Canadian Geotechnical Journal 47, No. 8, 842-856.

Wang, D., Randolph, M. F. \& Gourvenec, S. (2015). Coefficient of consolidation for soil that elusive quantity. VI International Conference on Coupled Problems in Science and Engineering, 18-20 May 2015, Venice, Italy.

White, D. J., Gaudin, C., Boylan, N. \& Zhou, H. (2010). Interpretation of T-bar penetrometer tests at shallow embedment and in very soft soils. Canadian Geotechnical Journal 47, No. 2, 218-229.

White D.J., Hill A.J., Westgate Z. \& Ballard J-C (2011). Observations of pipe-soil response from the first deepwater deployment of the SMARTPIPE. Proc. 2nd Int. Symp. on Frontiers in Offshore Geotechnics. Perth. 851-856

Whittle, A.J., Sutabutr, T., Germaine, J.T. \& Varney, A. (2001). Prediction and interpretation of pore pressure dissipation for a tapered piezoprobe. Geotechnique, 51, No. 7, 601617.

Wood, D. M. (1990). Soil behaviour and critical state soil mechanics. Cambridge university press. 
Schneider, M. A., Stanier, S. A., Chatterjee, S., White, D. J. and Randolph, M. F.

The parkable piezoprobe for determining $c_{v}$ and strength - modelling and interpretation methods

Zienkiewicz, O. C. \& Zhu, J. Z. (1992). The superconvergent patch recovery and a posteriori error estimates. Part 1: The recovery technique. International Journal for Numerical Methods in Engineering 33, No. 7, 1331-1364. 
Schneider, M. A., Stanier, S. A., Chatterjee, S., White, D. J. and Randolph, M. F.

The parkable piezoprobe for determining $c_{v}$ and strength - modelling and interpretation methods

\section{APPENDIX}

The LDFE code developed to generate the simulations presented in this manuscript were first benchmarked against results published by Chatterjee et al. (2014) using the geometries and soil parameters outlined in that paper, before exploring the effects of finite element mesh density, penetration rate, surface drainage assumption and permeability function.

Figure 15 presents results from a simulation with a voids ratio dependent permeability function (after Mahmoodzadeh et al. 2014) and a simulation with a constant permeability $(k=$ 1e-9 $\mathrm{m} / \mathrm{s}$ ). There is a relatively small difference between the simulations for the normally consolidated conditions modelled in this benchmarking exercise, however, both are in close agreement with the result published by Chatterjee et al. (2014). This indicates that the LDFE code developed is robust and consistent with that used in previous work and that the difference in permeability distributions modelled (isotropic permeability vs. permeability derived from voids ratio) has minimal impact on the undrained penetration resistance.

Figure 16 presents results for two free surface pore pressure assumptions: that used in this study where the free surface pore pressure was elevation dependent and another where it was assumed to always be zero (as in Chatterjee et al. 2014). There is a small increase in penetration resistance for the case with elevation dependent surface pore pressure, which is due to local heave leading to a pressure differential that increases the drainage rate within the heaving material surrounding the penetrometer.

Figure 17 presents the results of the mesh sensitivity analysis. The coarse mesh gives a higher penetration resistance, whereas the mesh used in this study and the finer mesh show no discernible variation in response, therefore validating the use of the mesh used throughout the current study.

Figure 18 presents the results of simulations with varying non-dimensional penetration velocity $\left(V=v D / c_{v}\right)$ over the range of 50-150 with 100 being the value used throughout this 
Schneider, M. A., Stanier, S. A., Chatterjee, S., White, D. J. and Randolph, M. F.

The parkable piezoprobe for determining $c_{v}$ and strength - modelling and interpretation methods

study. There is a significant reduction in non-dimensional penetration resistance when $V$ is increased from 50 to 100 (> 5\%); however, in contrast, when $V$ is increased further to 150 the reduction is far less significant $(<1 \%)$. This illustrates that a non-dimensional penetration velocity of 100 is sufficiently high to ensure minimal dissipation of excess pore pressures during penetration. 
Schneider, M. A., Stanier, S. A., Chatterjee, S., White, D. J. and Randolph, M. F.

The parkable piezoprobe for determining $c_{v}$ and strength - modelling and interpretation methods

\section{FIGURES}

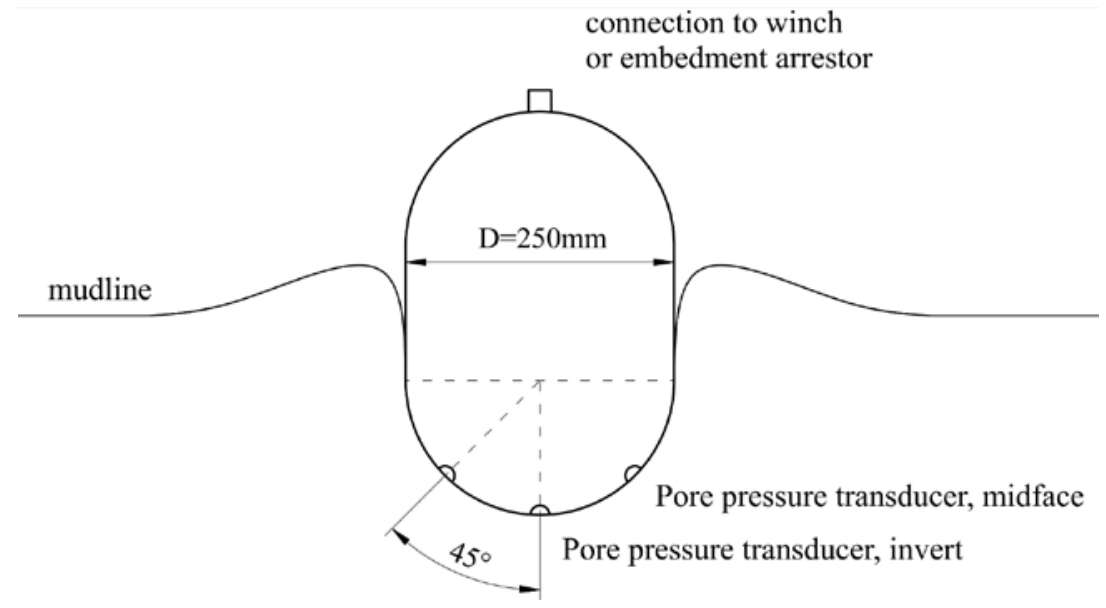

(a)

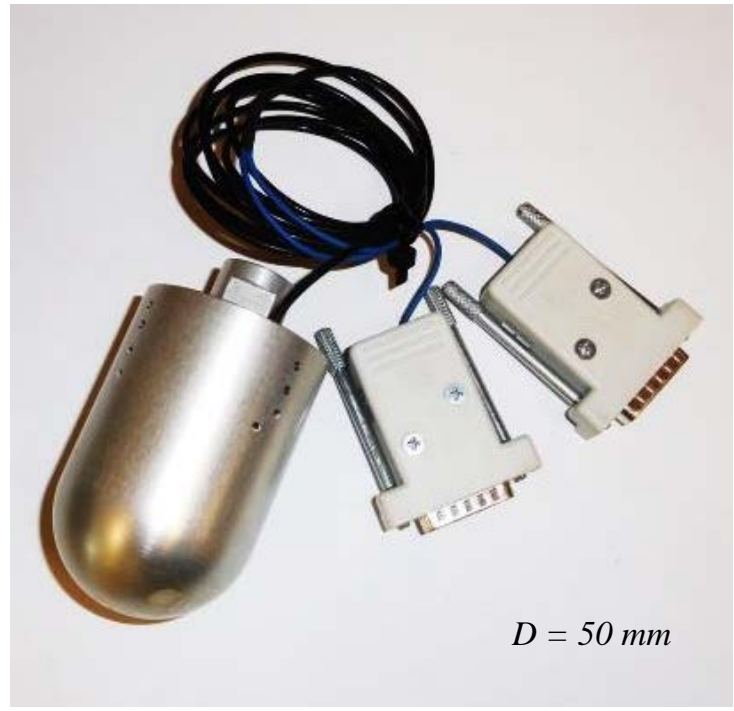

(b)

Figure 1: (a) Illustration of parkable piezoprobe; (b) and small-scale centrifuge model. 
Schneider, M. A., Stanier, S. A., Chatterjee, S., White, D. J. and Randolph, M. F.

The parkable piezoprobe for determining $c_{v}$ and strength - modelling and interpretation methods

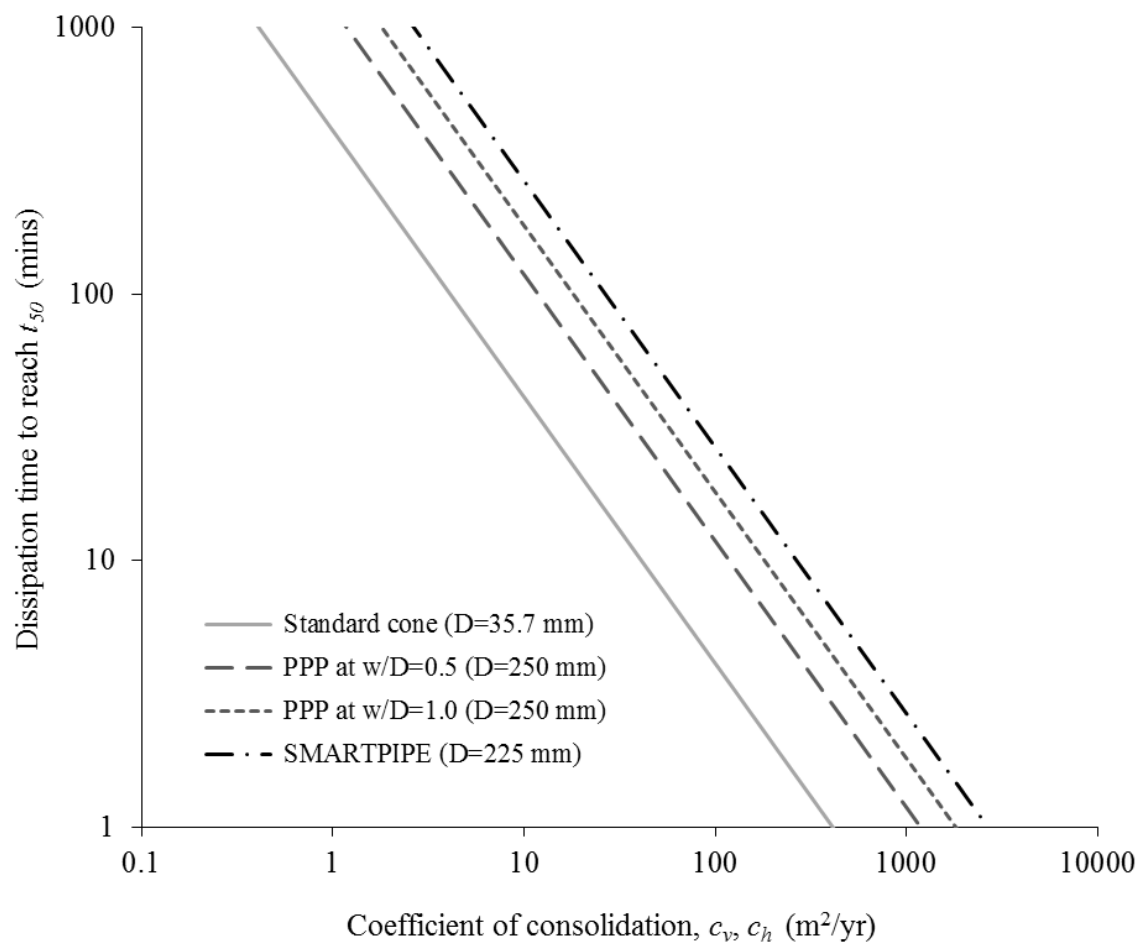

Figure 2: Dimensional time required for $50 \%$ of excess pore pressures to dissipate, $t_{50}$, for the PPP, cone penetrometer and SMARTPIPE.

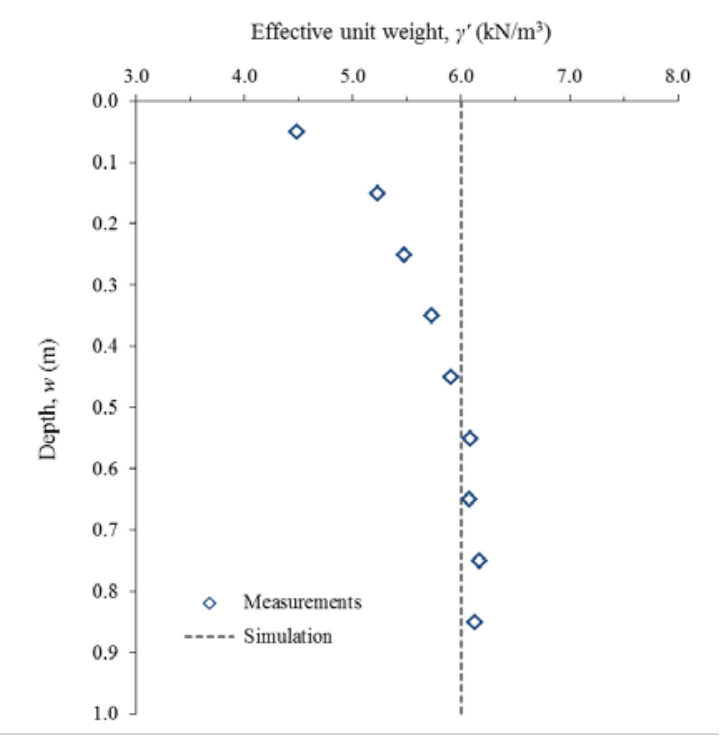

(a)

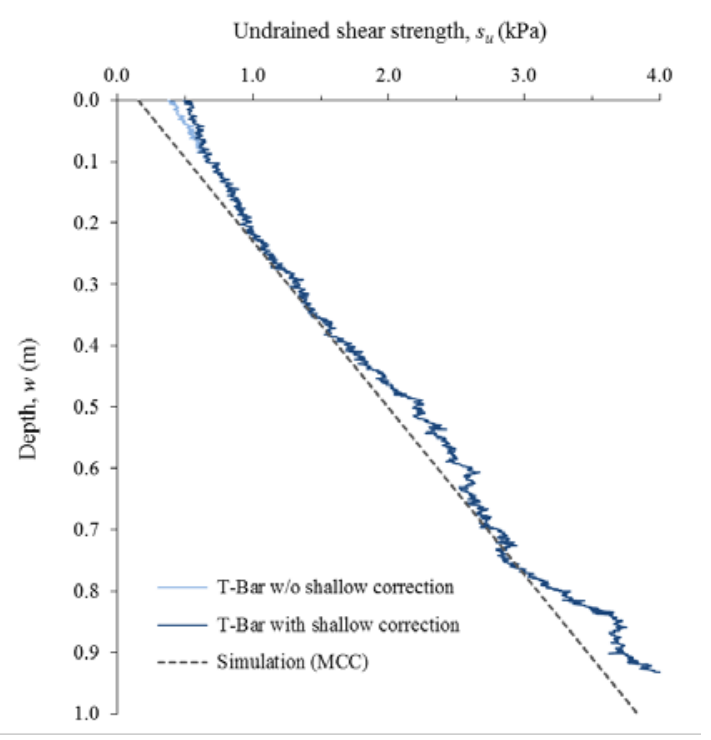

(b)

Figure 3: Soil properties measured vs. modelled: (a) effective unit weight; and (b) undrained shear strength. 
Schneider, M. A., Stanier, S. A., Chatterjee, S., White, D. J. and Randolph, M. F.

The parkable piezoprobe for determining $c_{v}$ and strength - modelling and interpretation methods

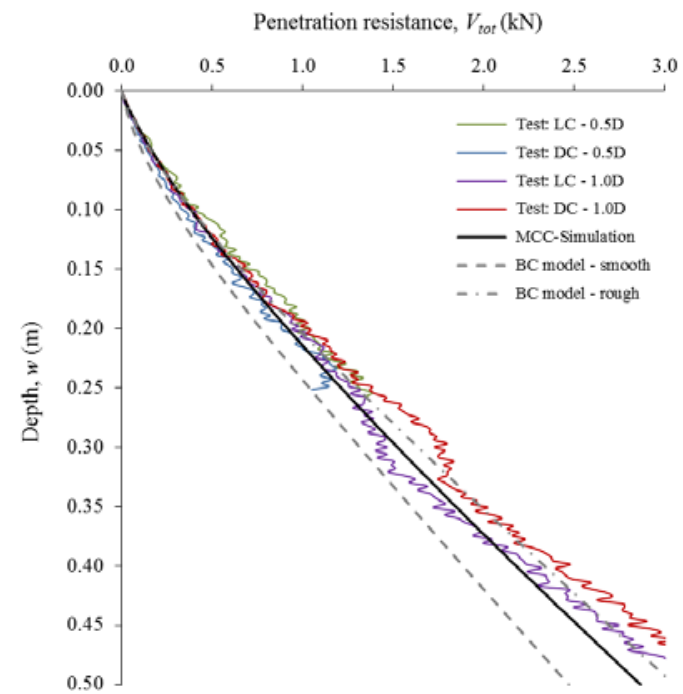

(a)

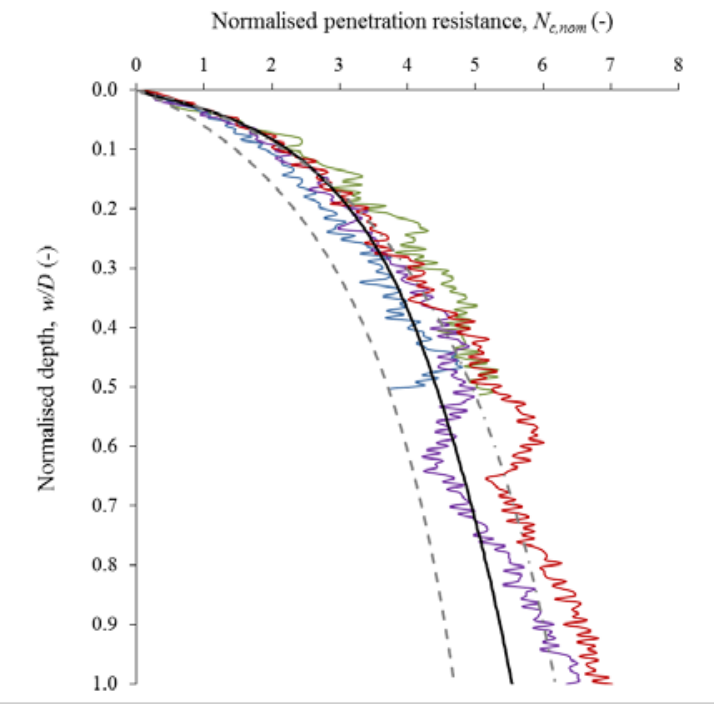

(b)

Figure 4: Comparison of experimental measurements and numerical simulation: (a) total penetration resistance vs. depth; and (b) normalised penetration resistance vs. normalised depth. 
Schneider, M. A., Stanier, S. A., Chatterjee, S., White, D. J. and Randolph, M. F.

The parkable piezoprobe for determining $c_{v}$ and strength - modelling and interpretation methods

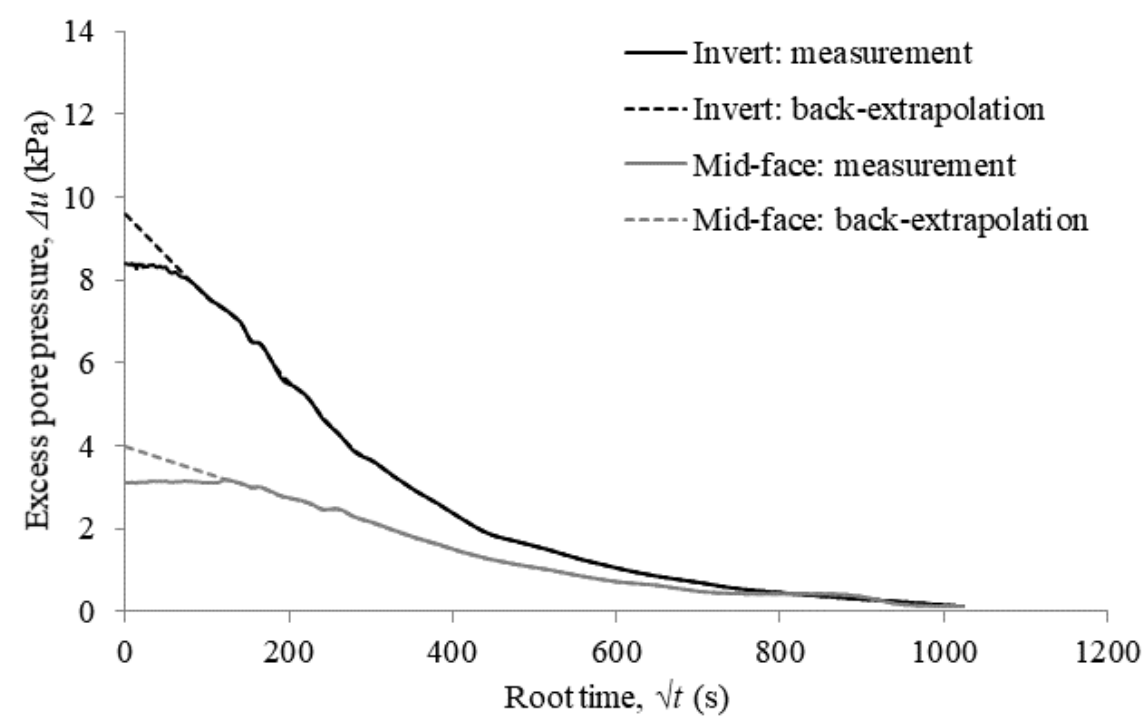

(a)

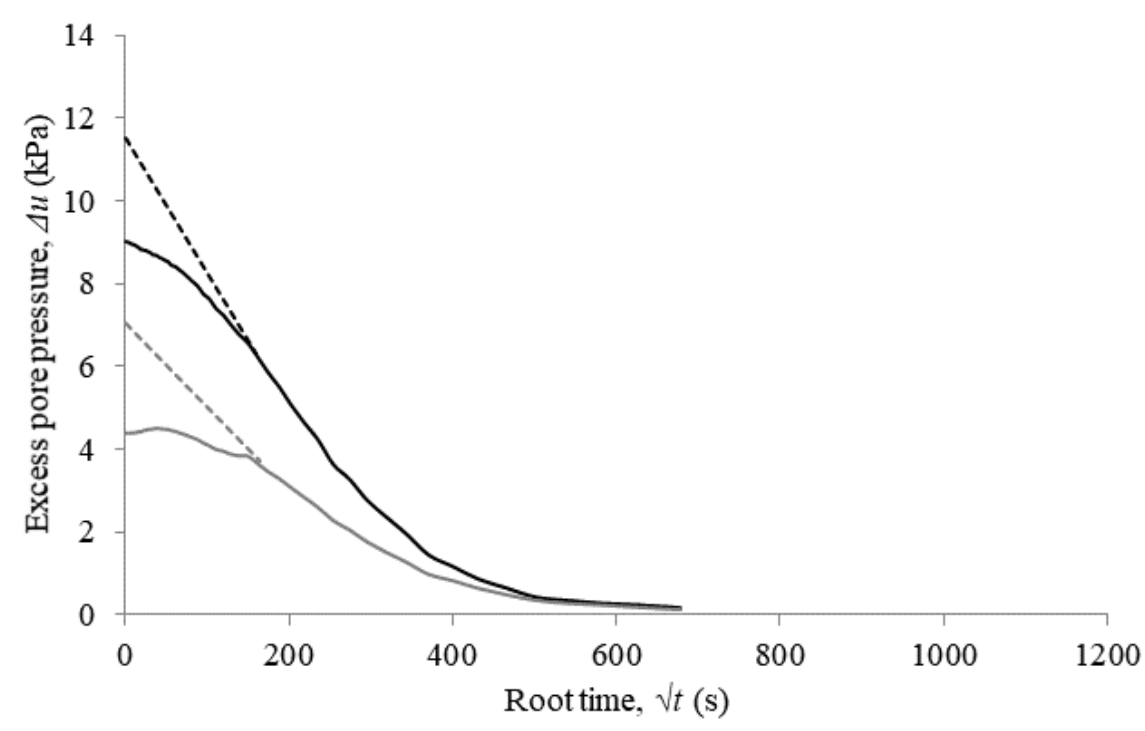

(b) 
Schneider, M. A., Stanier, S. A., Chatterjee, S., White, D. J. and Randolph, M. F.

The parkable piezoprobe for determining $c_{v}$ and strength - modelling and interpretation methods

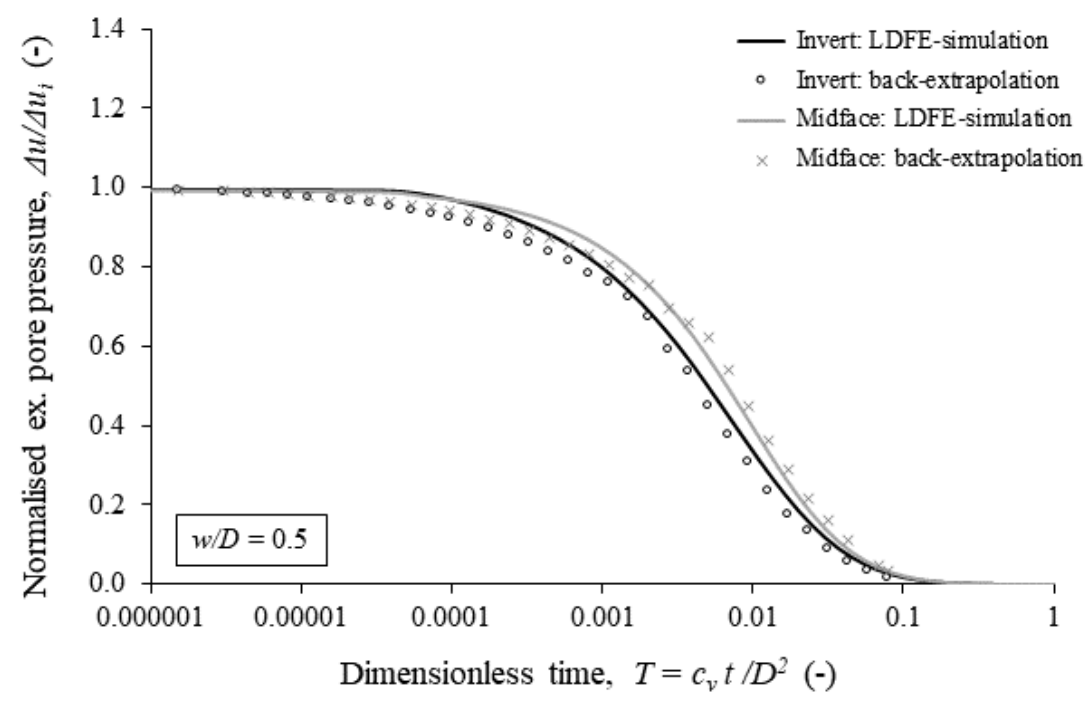

1

(c)

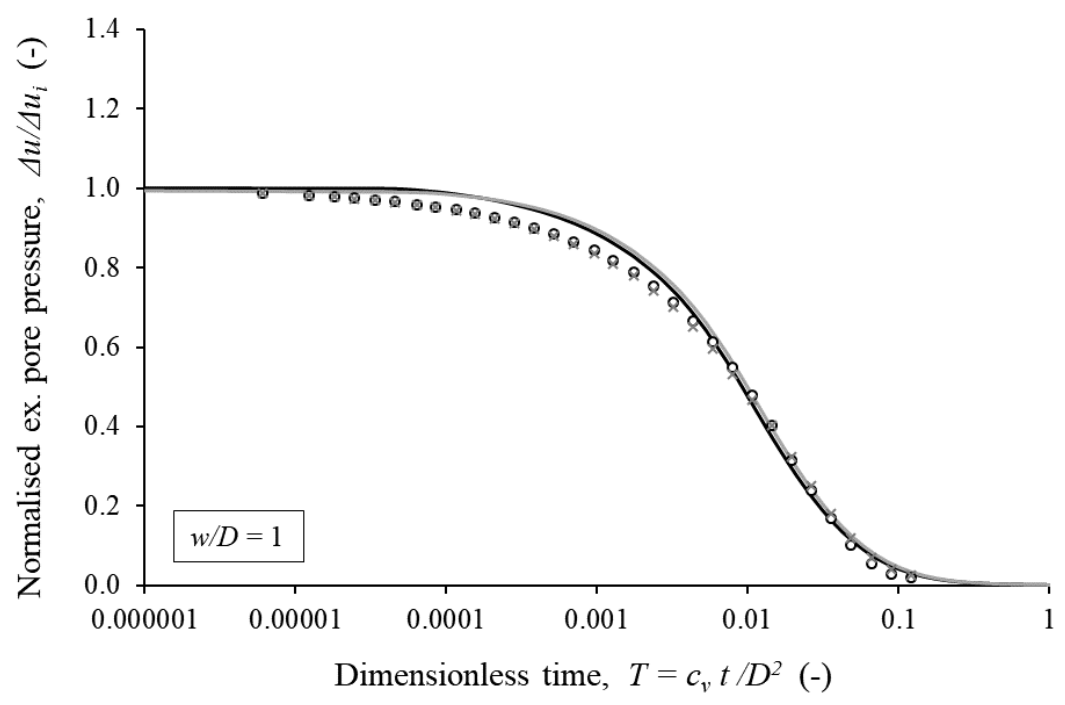

(d)

Figure 5: Experimental measurements with and without the Sully et al. (1999) correction compared to the numerical simulations: (a, b) dimensional response; and (c, d) non-dimensional response for $w / D$ of 0.5 and 1 , respectively. 
Schneider, M. A., Stanier, S. A., Chatterjee, S., White, D. J. and Randolph, M. F.

The parkable piezoprobe for determining $c_{v}$ and strength - modelling and interpretation methods

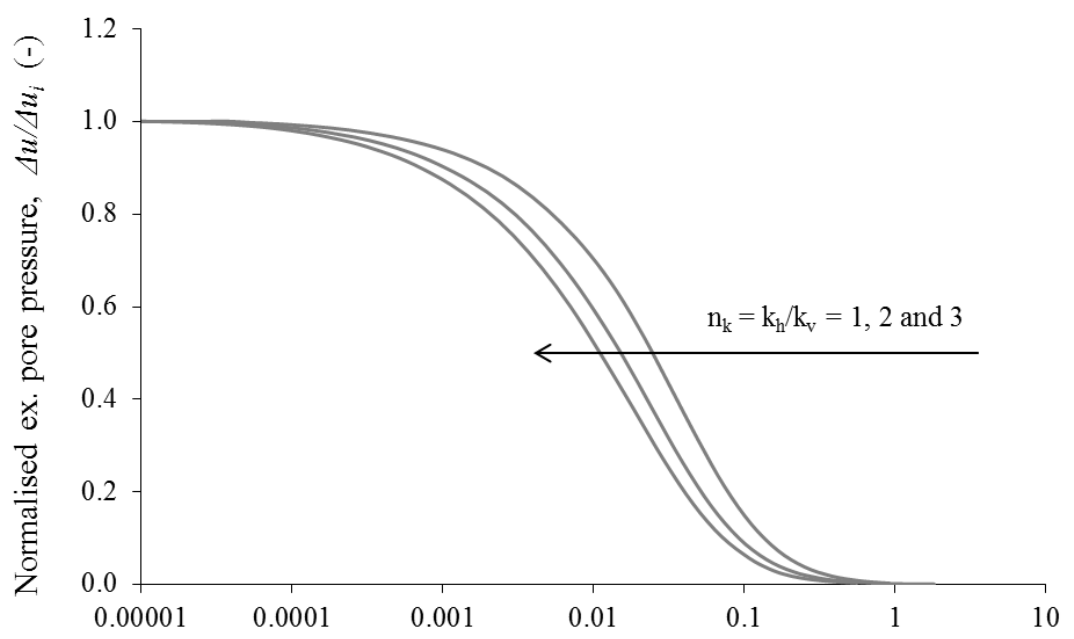

Dimensionless time, $T=c_{v 0} t / D^{2}(-)$

(a)

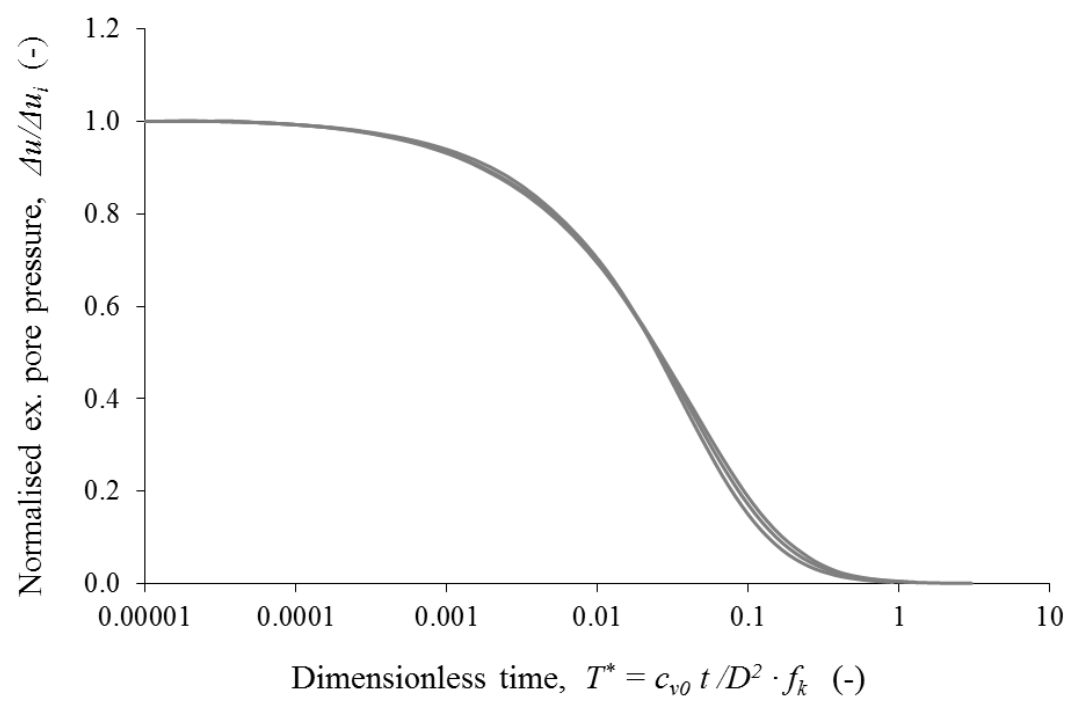

(b)

Figure 6: Effect of $n_{k}$ on normalised dissipation response at $w / D=0.5$ : (a) conventional normalisation; and (b) proposed normalisation. 
Schneider, M. A., Stanier, S. A., Chatterjee, S., White, D. J. and Randolph, M. F.

The parkable piezoprobe for determining $c_{v}$ and strength - modelling and interpretation methods

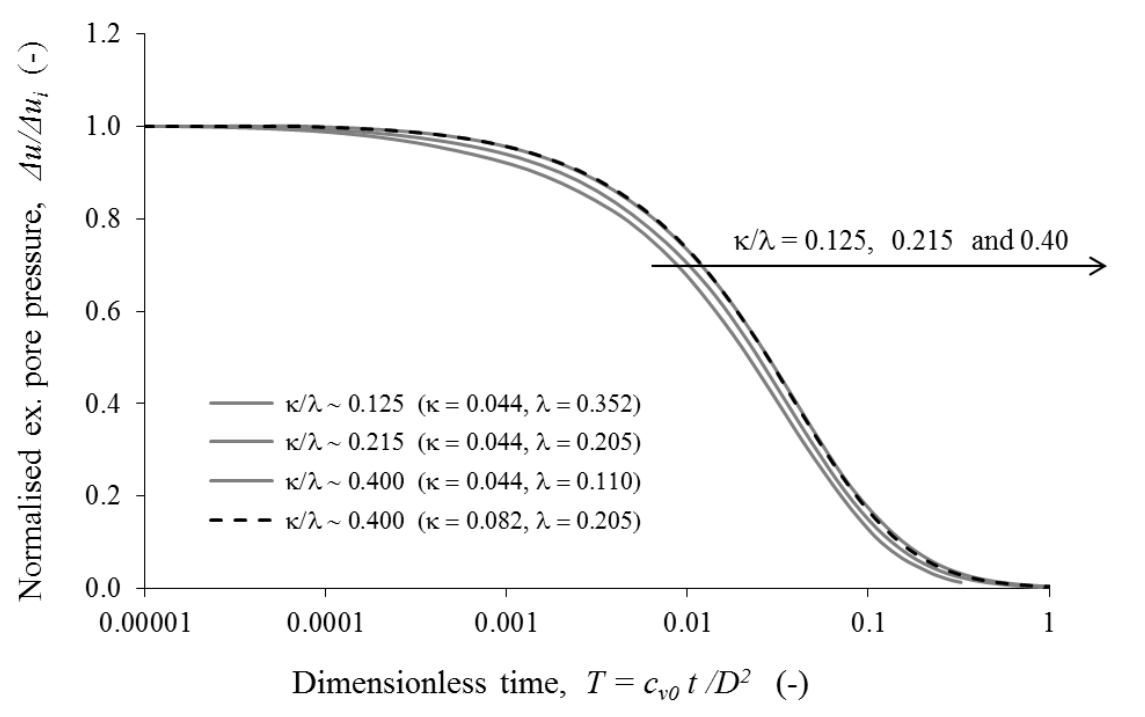

(a)

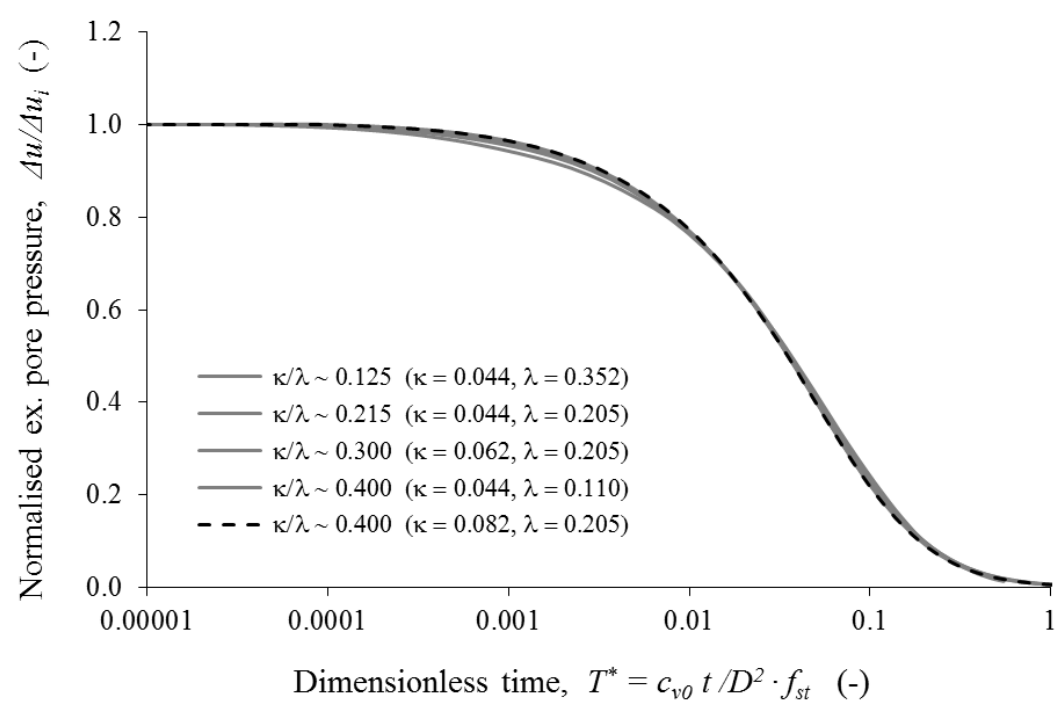

(b)

Figure 7: Effect of ratio $\kappa / \lambda$ on normalised dissipation response at $w / D=0.5$ : (a) conventional normalisation; and (b) proposed normalisation. 
Schneider, M. A., Stanier, S. A., Chatterjee, S., White, D. J. and Randolph, M. F.

The parkable piezoprobe for determining $c_{v}$ and strength - modelling and interpretation methods

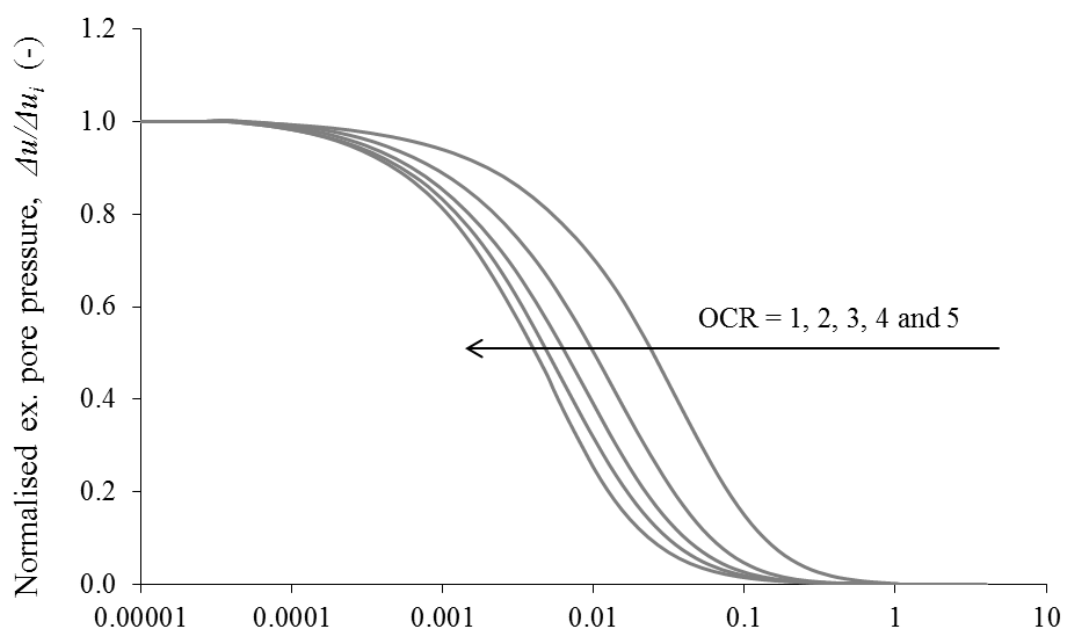

Dimensionless time, $T=c_{v 0} t / D^{2}(-)$

(a)

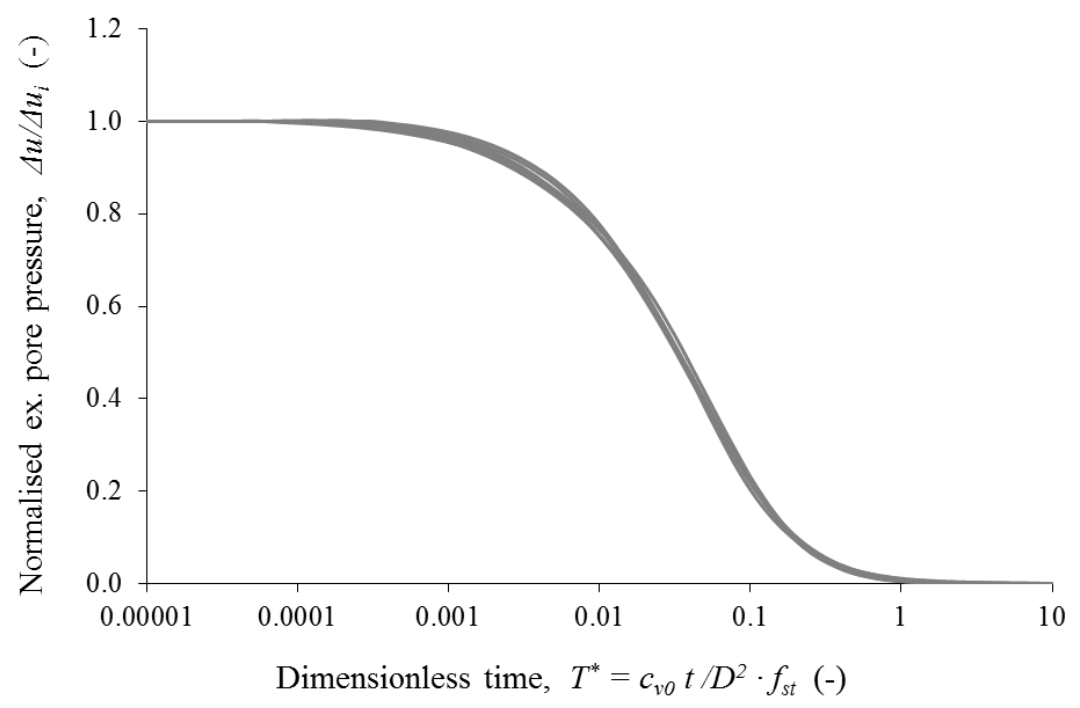

(b)

Figure 8: Effect of OCR on normalised dissipation response at $w / D=0.5$ : (a) conventional normalisation; and (b) proposed normalisation. 
Schneider, M. A., Stanier, S. A., Chatterjee, S., White, D. J. and Randolph, M. F.

The parkable piezoprobe for determining $c_{v}$ and strength - modelling and interpretation methods

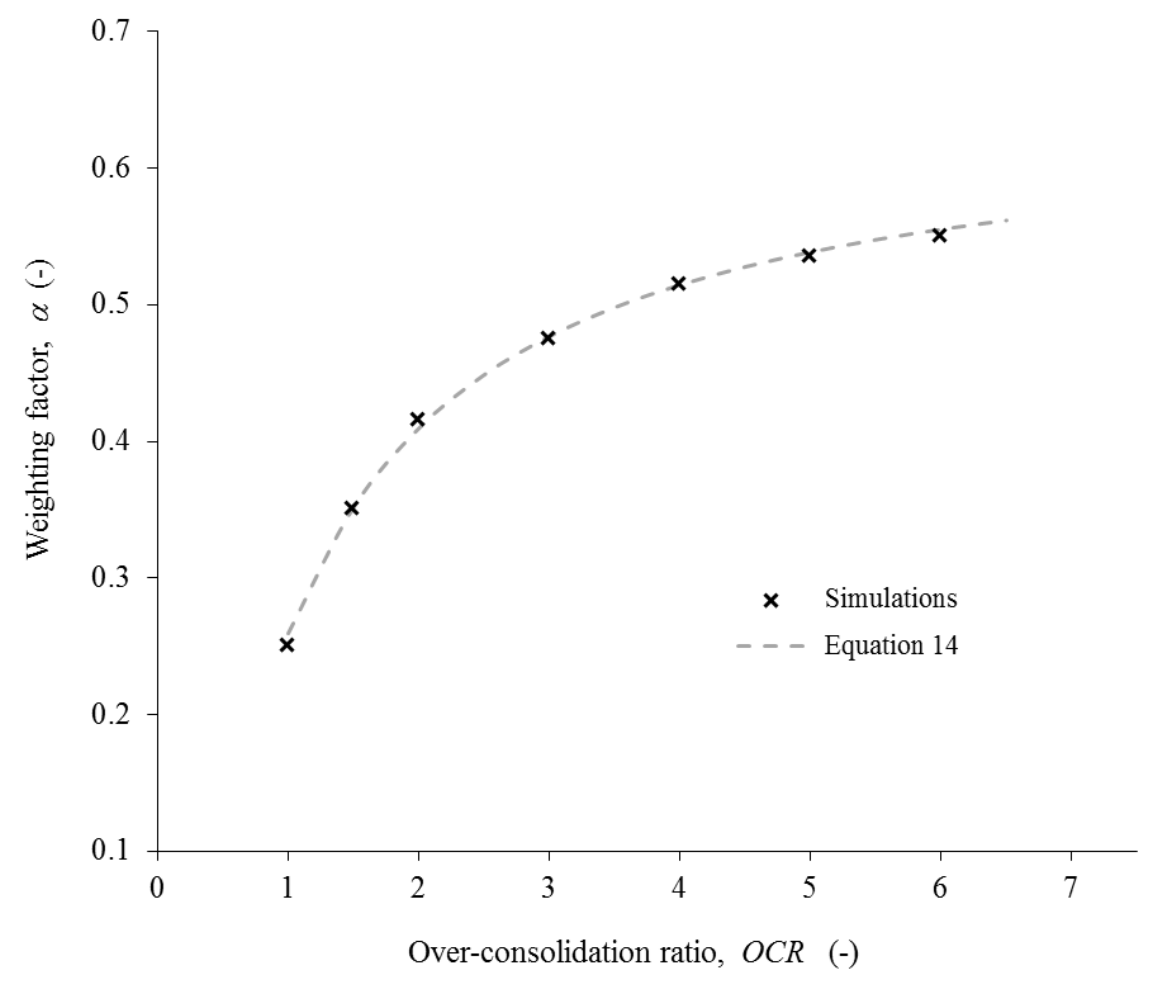

Figure 9: Definition of weighting factor as a function of $O C R$.

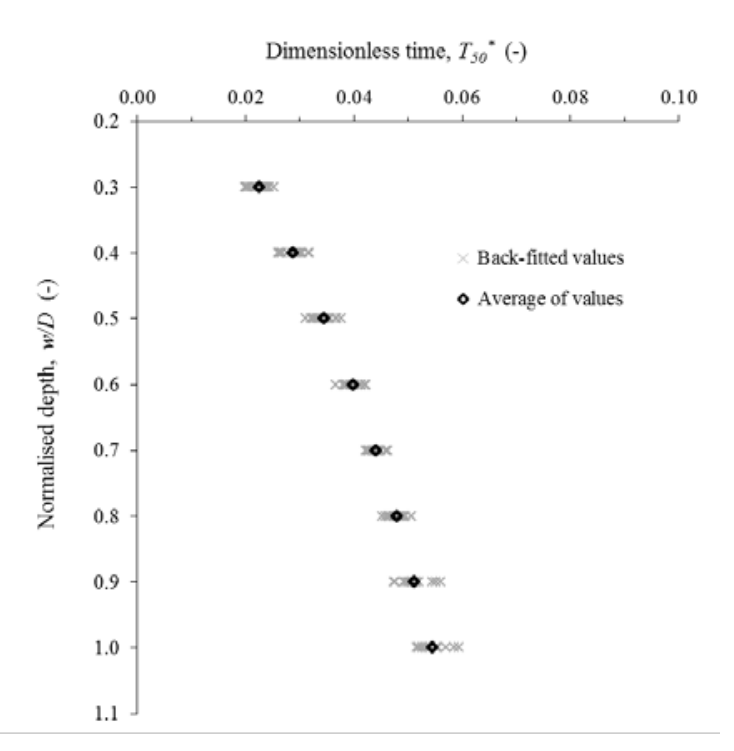

(a)

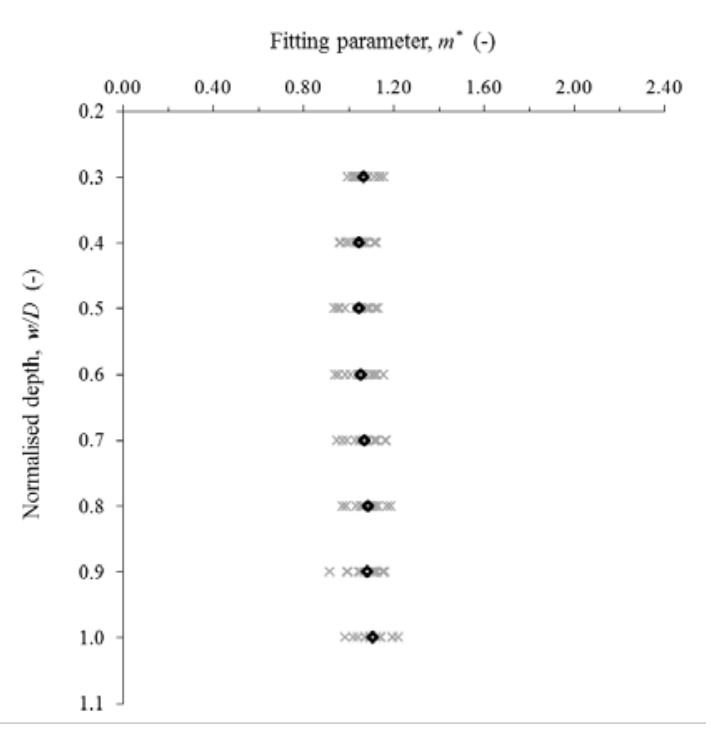

(b)

Figure 10: Dependency on embedment depth of back-fitted: (a) $T_{50} *$ and (b) $m *$ for invert position. 
Schneider, M. A., Stanier, S. A., Chatterjee, S., White, D. J. and Randolph, M. F.

The parkable piezoprobe for determining $c_{v}$ and strength - modelling and interpretation methods

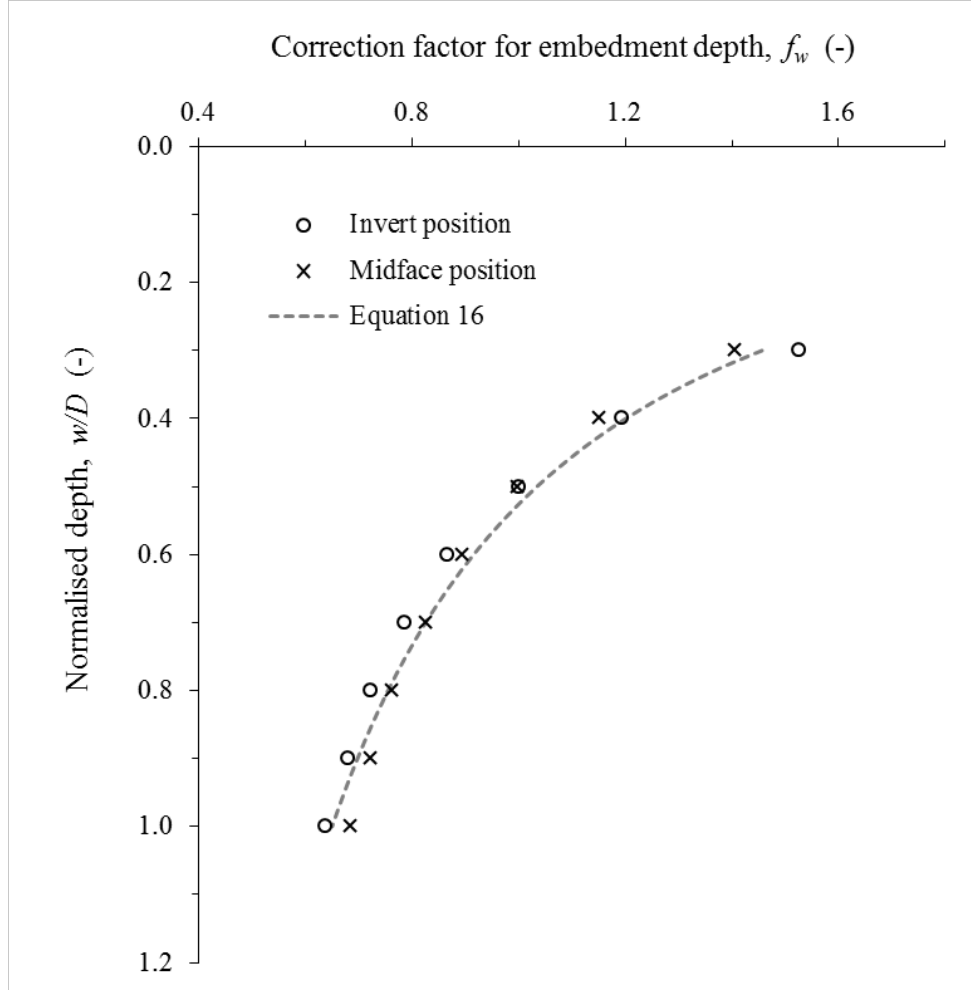

Figure 11: Definition of embedment influence factor for invert and midface location

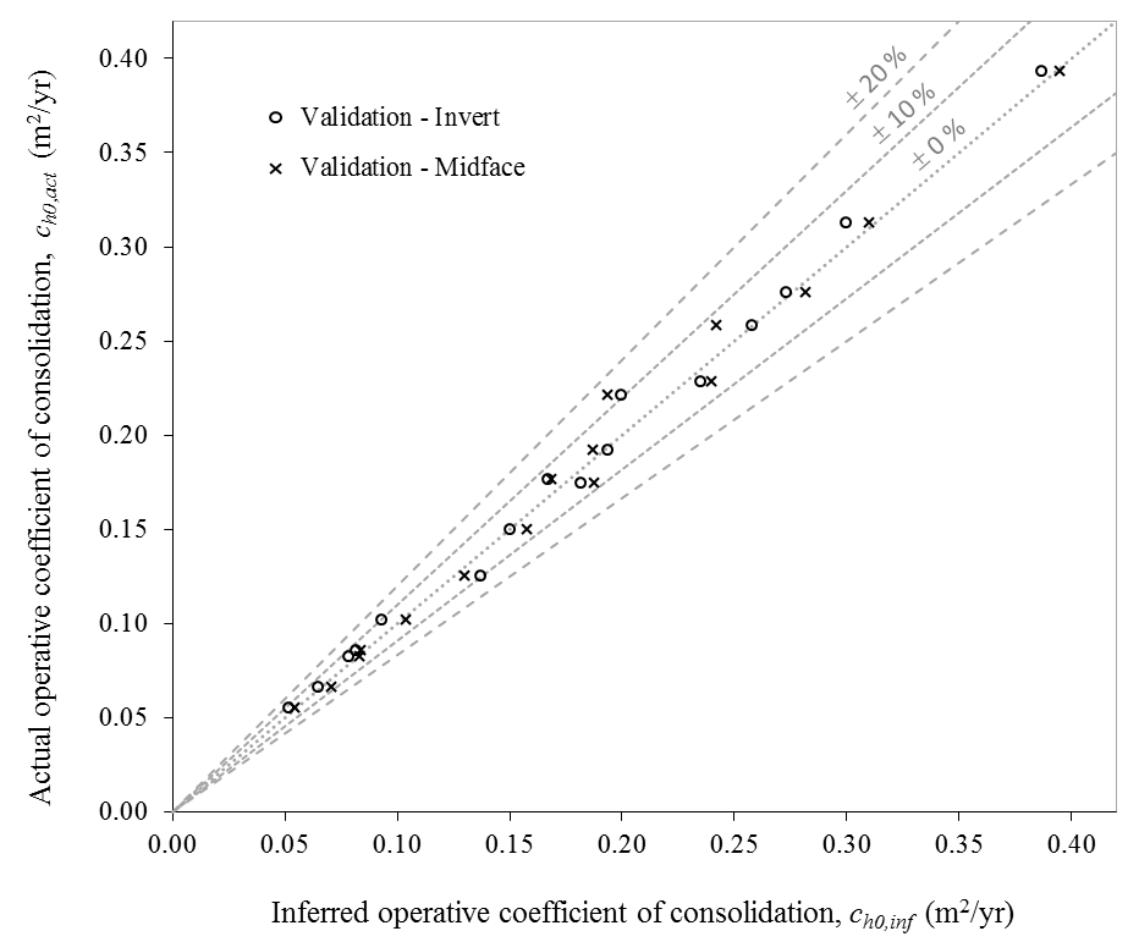

Figure 12: Performance and validation of proposed interpretation-model. 
Schneider, M. A., Stanier, S. A., Chatterjee, S., White, D. J. and Randolph, M. F.

The parkable piezoprobe for determining $c_{v}$ and strength - modelling and interpretation methods

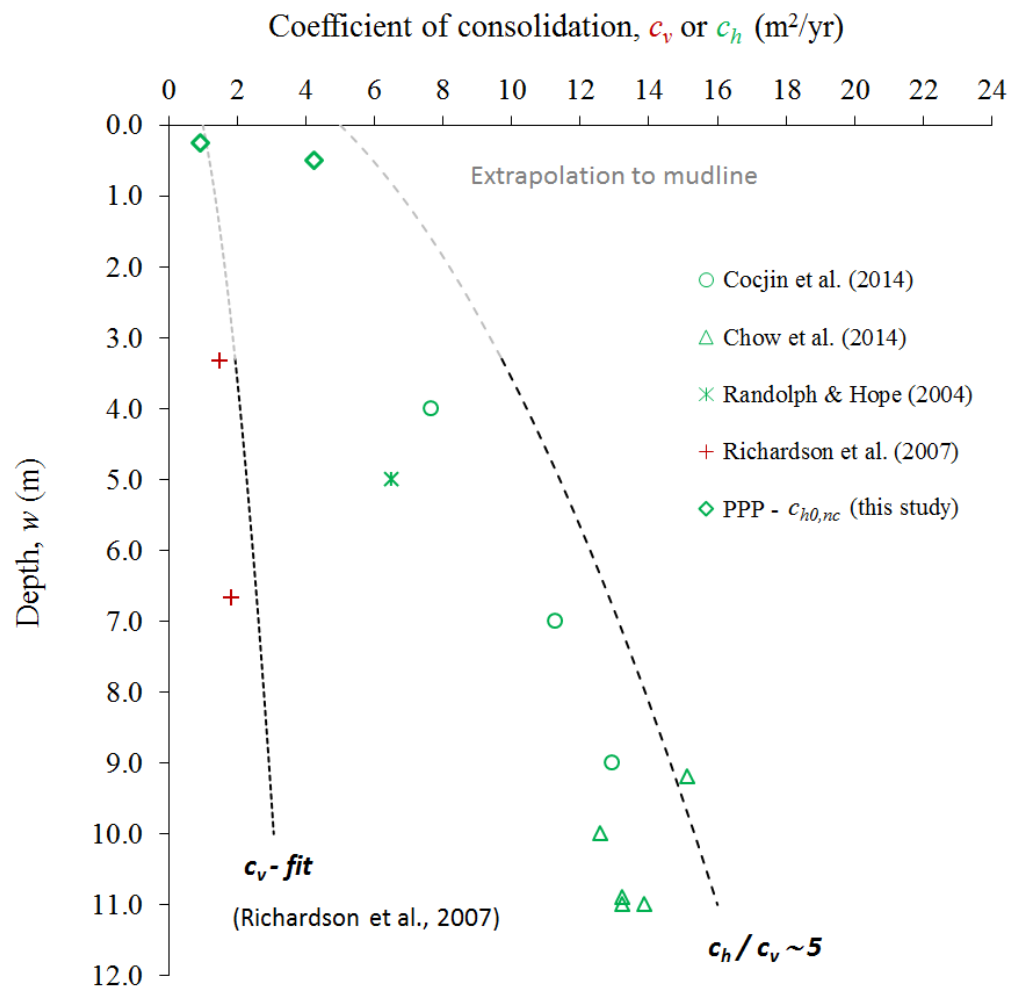

Figure 13: Inferred $c_{h}$-values of centrifuge experiments compared to values given in literature.

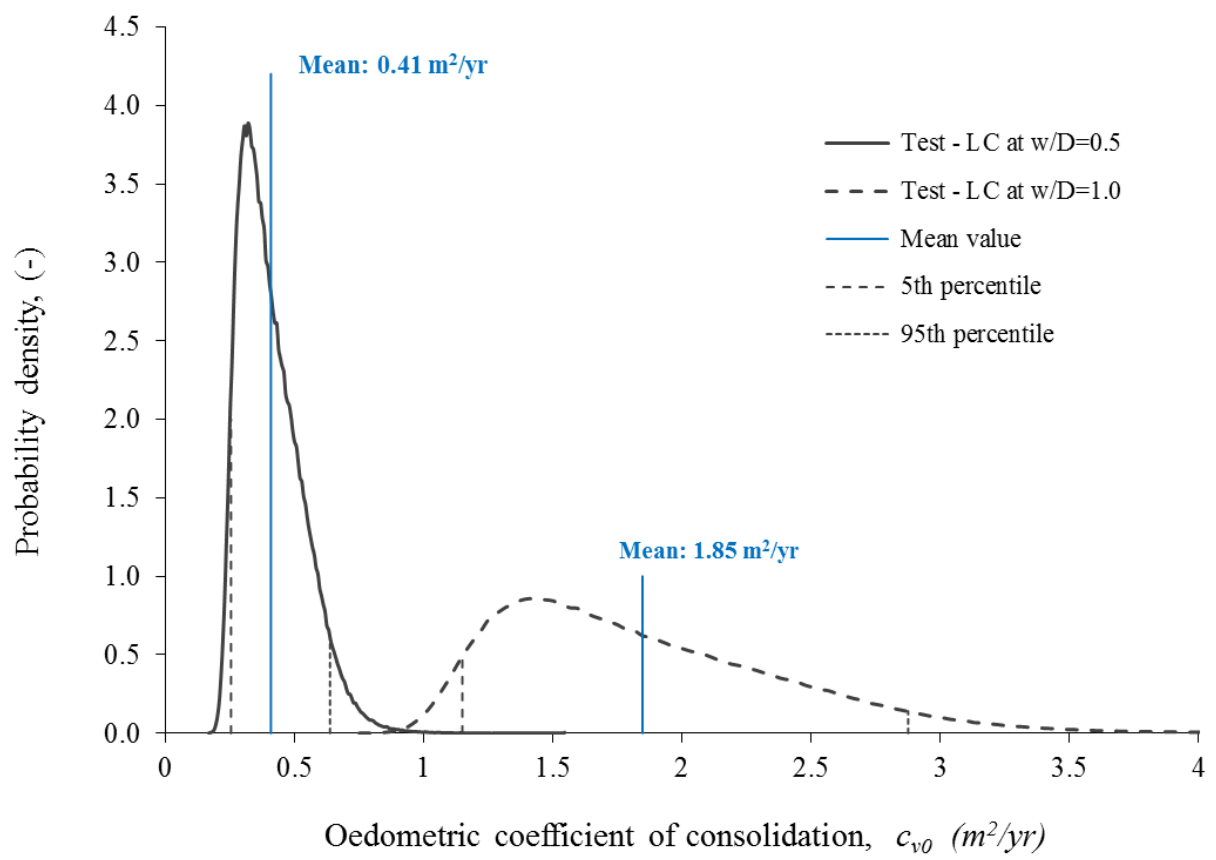

Figure 14: Estimated distributions of oedometric $c_{v 0}$ values (Monte Carlo simulation). 
Schneider, M. A., Stanier, S. A., Chatterjee, S., White, D. J. and Randolph, M. F.

The parkable piezoprobe for determining $c_{v}$ and strength - modelling and interpretation methods

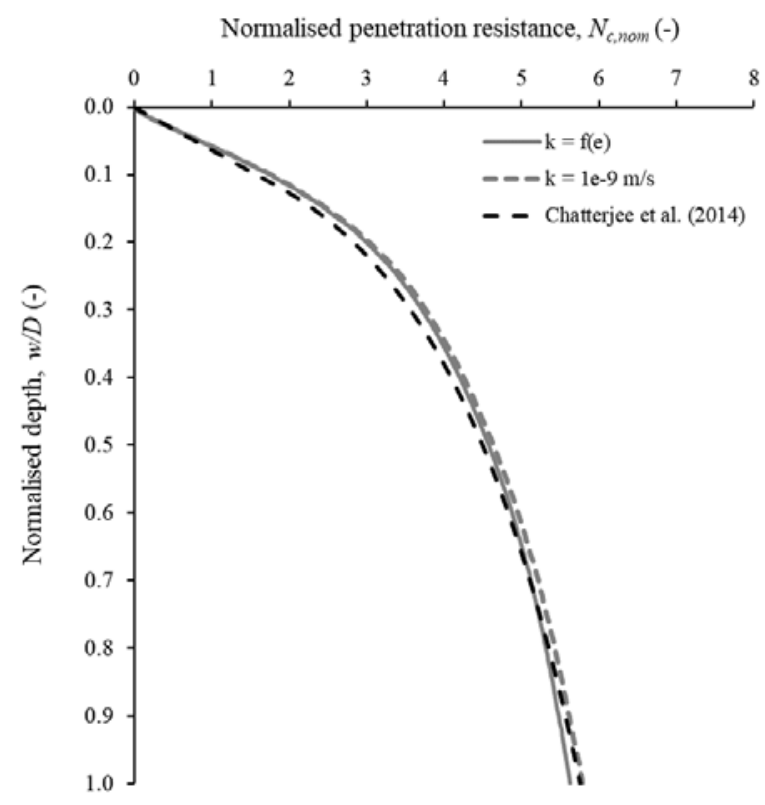

Figure 15: Comparison of results from the current manuscript with a voids ratio dependent permeability function with simulations with a constant permeability assumption and published simulation results after Chatterjee et al. (2014).

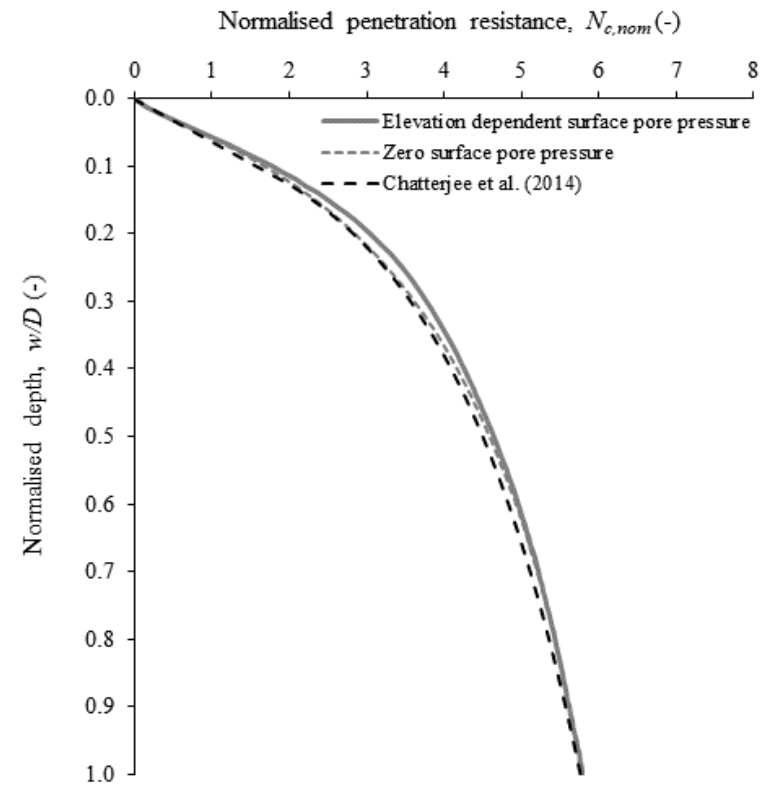

Figure 16: Comparison of results from the current manuscript with an elevation dependent pore pressure assumption with simulations with a zero surface pore pressure assumption and published simulation results after Chatterjee et al. (2014). 
Schneider, M. A., Stanier, S. A., Chatterjee, S., White, D. J. and Randolph, M. F.

The parkable piezoprobe for determining $c_{v}$ and strength - modelling and interpretation methods

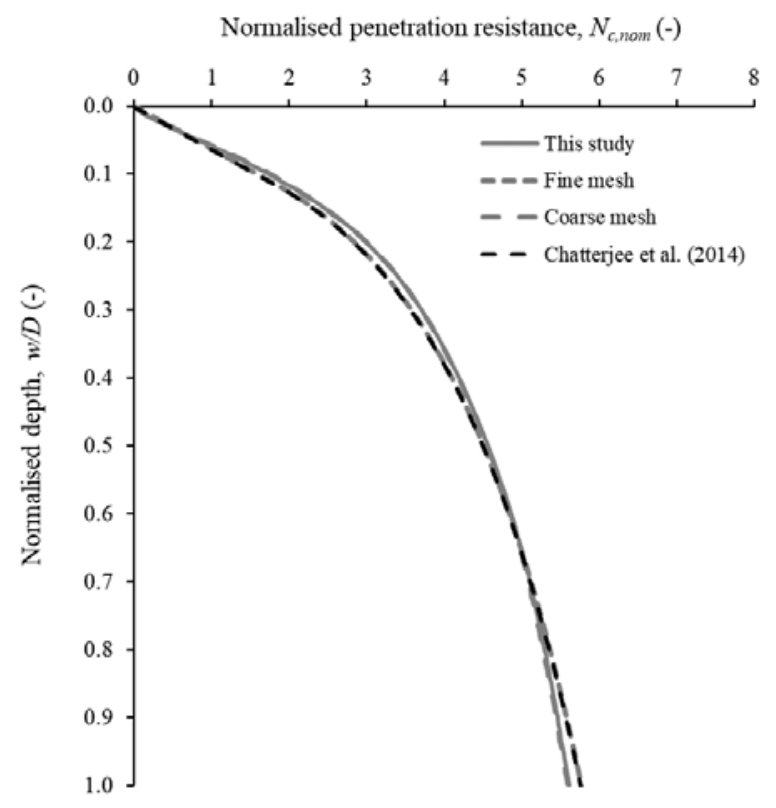

Figure 17: Comparison of results from the current manuscript using a finite element mesh with 18,105 elements with simulations with finer (31,288 elements) and coarser (12,217 elements) meshes and published simulation results after Chatterjee et al. (2014).

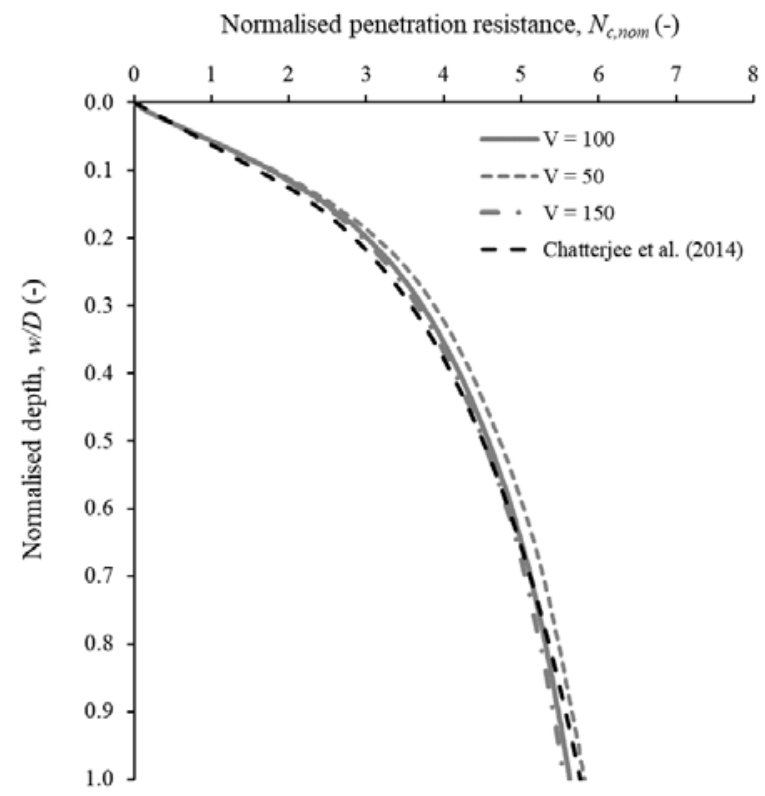

Figure 18: Comparison of results from the current manuscript where non-dimensional penetration velocity $V\left(=v D / c_{v}\right)$ was 100 with simulations with $V$ of 50 and 100 and published simulation results after Chatterjee et al. (2014). 